\title{
Effects of rotation and sloping terrain on the fronts of density currents
}

\author{
By J. C. R. HUNT ${ }^{1,2}$, J. R. PACHECO ${ }^{2}$, A. MAHALOV $\mathrm{MAC}^{3}$, \\ AND H. J. S. FER N A N D ${ }^{2}$
}

\author{
${ }^{1}$ Departments of Space and Climate Physics and Earth Sciences, University College London, \\ London, WC1H 6BT, UK and J. M. Burgers Centre, Delft University of Technology, Delft, Netherlands \\ ${ }^{2}$ Department of Mechanical and Aerospace Engineering, Arizona State University, Tempe, \\ AZ 85287-6106, USA \\ ${ }^{3}$ Department of Mathematics, Arizona State University, Tempe, AZ 85287, USA
}

(Received 8 October 2003 and in revised form 11 March 2005)

The initial stage of the adjustment of a gravity current to the effects of rotation with angular velocity $f / 2$ is analysed using a short time analysis where Coriolis forces are initiated in an inviscid von Kármán-Benjamin gravity current front at $t_{F}=0$. It is shown how, on a time-scale of order $1 / f$, as a result of ageostrophic dynamics, the slope and front speed $U_{F}$ are much reduced from their initial values, while the transverse anticyclonic velocity parallel to the front increases from zero to $O\left(\mathrm{NH}_{0}\right)$, where $N=\sqrt{g^{\prime} / H_{0}}$ is the buoyancy frequency, and $g^{\prime}=g \Delta \rho / \rho_{0}$ is the reduced acceleration due to gravity. Here $\rho_{0}$ is the density and $\Delta \rho$ and $H_{0}$ are the density difference and initial height of the current. Extending the steady-state theory to account for the effect of the slope $\sigma$ on the bottom boundary shows that, without rotation, $U_{F}$ has a maximum value for $\sigma=\pi / 6$, while with rotation, $U_{F}$ tends to zero on any slope. For the asymptotic stage when $f t_{F} \gg 1$, the theory of unsteady waves on the current is reviewed using nonlinear shallow-water equations and the van der Pol averaging method. Their motions naturally split into a 'balanced' component satisfying the Margules geostrophic relation and an equally large 'unbalanced' component, in which there is horizontal divergence and ageostrophic vorticity. The latter is responsible for nonlinear oscillations in the current on a time scale $f^{-1}$, which have been observed in the atmosphere and field experiments. Their magnitude is mainly determined by the initial potential energy in relation to that of the current and is proportional to the ratio $\sqrt{B u}=L_{R} / R_{0}$, where $L_{R}=N H_{0} / f$ is the Rossby deformation radius and $R_{0}$ is the initial radius. The effect of slope friction also prevents the formation of a steady front. From the analysis it is concluded that a weak mean radial flow must be driven by the ageostrophic oscillations, preventing the mean front speed $U_{F}$ from halting sharply at $f t_{F} \sim 1$. Depending on the initial value of $L_{R} / R_{0}$, physical arguments show that $U_{F}$ decreases slowly in proportion to $\left(f t_{F}\right)^{-1 / 2}$, i.e. $U_{F} / U_{F_{0}}=F\left(f t_{F}, B u\right)$. Thus the front only tends to the geostrophic asymptotic state of zero radial velocity very slowly (i.e. as $f t_{F} \rightarrow \infty$ ) for finite values of $L_{R} / R_{0}$. However, as $L_{R} / R_{0} \rightarrow 0$, it reaches this state when $f t_{F} \sim 1$. This analysis of the overall nonlinear behaviour of the gravity current is consistent with two two-dimensional non-hydrostatic (Navier-Stokes) and axisymmetric hydrostatic (shallow-water) Eulerian numerical simulations of the varying form of the rotating gravity current. When the effect of surface friction is considered, it is found that the mean movement of the front is significantly slowed. Furthermore, the oscillations with angular frequency $f$ and the slow growth of the radius, when $f t_{F} \geqslant 1$, are consistent with recent experiments. 


\section{Introduction}

Characteristic features of many geophysical flows, on a wide variety of length and time scales, are sloping interfaces that separate zones of less dense and more dense fluid. Because long waves on stable layers of fluid tend to travel faster the deeper the layer, it means that fluid far from the deep part of the zone tends to move towards the shallower leading-edge of the zone. This process tends to increase or maintain high gradients of density near the boundary, which becomes a sharply defined interface. The higher hydrostatic pressure at the base of the zone of dense fluid forces it to move into the lower part of the zone of less dense fluid, producing the well-known phenomena of gravity currents (Simpson 1997).

On the mesoscale (of the order of $10-100 \mathrm{~km}$ in the atmosphere), there may be external shear flows opposing the movement of the gravity current fronts (Rottman, Hunt \& Mercer 1985) or strong externally produced mixing (e.g. Linden \& Simpson 1989; Noh \& Fernando 1993). In both of these cases the fronts tend to take the appearance of a wedge with a low angle. On larger meso-synoptic scales, the Earth's rotation causes the front to slow down, even bringing it to a standstill as shown in the laboratory by Chia, Griffiths \& Linden (1982) and Ivey (1987). In this case a 'geostrophic front' develops, which differs from a non-rotating gravity current in that it has a lower slope. Also, significant motions parallel to the frontal surface are induced both within the current and outside it. The flows parallel to the frontal surface balance the hydrostatic pressure gradients and Coriolis forces, and may lead to a stationary form of geostrophic front, as first explained by Margules (1906). However, there is no theory to show $(a)$ that this equilibrium state is always the limiting solution for density currents that are initially not in equilibrium and, if so, whether (b) the limit is independent of the initial conditions, (c) whether the steady state is reached in a finite period of time and distance from initial release, $(d)$ whether it is reached monotonically and $(e)$ how viscosity, turbulence and surface friction affect its behaviour. Some gravity currents move over fluid layers, which may also affect frontal dynamics. Such questions may be useful to meteorologists who are generally sceptical about the relevance of equilibrium geostrophic fronts to real atmospheric flows.

Experiments by Hallworth, Huppert \& Ungarish (2001) show how the radial spreading of an axisymmetric gravity current affected by Coriolis forces slows rapidly over a rotation period and then oscillates. It is not clear whether the mean radius of the current reaches a fixed value or continues to increase slowly. In their hydrostatic analysis, they successfully account for the initial stages of the gravity current's transformation into a geostrophic front, but they assumed for simplicity that the speed of the current's front was unaffected by rotation, although this is not valid when a front becomes a geostrophic front. Frontal dynamics tends to be discussed, especially by theoretical meteorologists and oceanographers, in terms of two distinct idealized models, namely, the density current which includes no representation of Coriolis effects and the balanced semi-geostrophic (SG) front which develops from larger-scale flows. For meteorological applications see Hoskins \& Bretherton (1972) and Parker (2000). Gravity currents and fronts on sloping surfaces are of particular significance in oceanography where rotation generates very strong flows along the slope. The controversial question here is whether or how such currents stop moving down the slope when rotation effects are present (e.g. Lentz \& Helfrich 2002).

Our first goal is to study how the form of a density current front develops when the effects of rotation are included. Although Rotunno (1983) reviewed how a longwave analysis explains many features of sea breeze gravity currents and the effects 


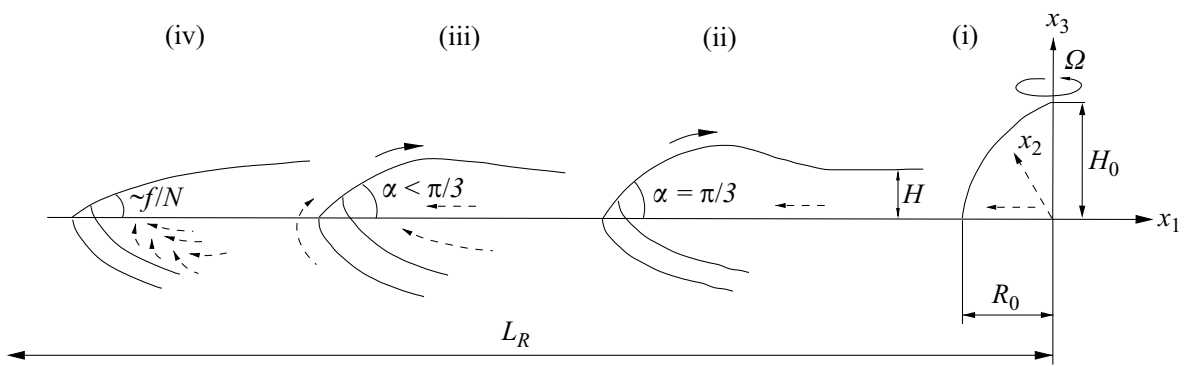

FiguRE 1. Typical stages in a density current front, with slope $\tan (\alpha)$, as it evolves towards that of a geostropic front. The Rossby radius of deformation $L_{R}$ is greater than the initial radius $R_{0}$ of the current. (i) Initial state; (ii) gravity current; (iii) evolving geostrophic front; (iv) geostrophic front. The dashed arrows denote velocity vectors in a rotating frame (but not moving with the front).

of rotation on them, he did not analyse the fronts themselves. His work, as with the earlier work of Benjamin (1968), showed that features of the current/frontal movements are largely independent of the details of the front. Our second goal is to study the evolution of the front when the flow is close to being in geostrophic balance, and when the effects of nonlinear ageostrophic oscillations are significant. In their daily task of forecasting the movement and evolution of fronts, meteorologists are well aware of the need to consider unsteady motions in fronts because of their effect on horizontal divergence and convergence, and on the mean motion of the front. Despite its dynamical significance, this ageostrophic component is not included in current models or even concepts of fronts (Parker 2000); for a review see Norbury \& Roulstone (2002).

The different stages and types of nonlinear adjustment of a front (see figure 1) can be classified in term of how the horizontal scale $R_{0}$ of initial disturbance of the dense layer relates to the Rossby radius of deformation $L_{R}$, which is defined as $L_{R}=\left(H_{0} g \Delta \rho / \rho_{0}\right)^{1 / 2} / f$ for two-layer systems (where $\Delta \rho$ is the density difference) and $L_{R}=N H_{0} / f$ in the case of a well-mixed region surrounded by fluid with continuous stratification ( $H_{0}$ is the depth of disturbance and $N$ is the buoyancy frequency). Our approach is to describe the flow regimes in terms of their nonlinear geostrophic adjustment to the equilibrium state, where initially $R_{0}<L_{R}, R_{0} \approx L_{R}$ or $R_{0}>L_{R}$. The Burgers number $B u=\left(L_{R} / R_{0}\right)^{2}$ which characterizes the relative importance of the effects of rotation and stratification (McWilliams 1985) plays a key role in distinguishing between different regimes, with $B u \ll 1$ corresponding to rotation dominated and $B u \gg 1$ corresponding to stratification dominated flows.

The geostrophic adjustment is relatively slow when $B u \gg 1$ and is only weakly coupled to 'quasi-inertial' oscillations with large horizontal and small vertical scale. Therefore these oscillations tend to persist, as is observed in the atmosphere and the ocean (Sidi \& Barat 1986; Maas \& van Haren 1987) and in the experiments of Hallworth et al. (2001). These two types of motion are studied here both computationally and theoretically.

By contrast in the regime where $B u \ll 1$, the characteristic sub-synoptic gravity waves and gravity currents adjust fast in the mean, and have a quasi-steady behaviour under the influence of rotational effects. Barotropic instabilities of the front are a significant feature of these flows (e.g. Linden \& Simpson 1989). This paper focuses on the various aspects of the unsteady nonlinear adjustment in the range of values 
$B u \gg 1$ to $B u=O(1)$ regime. Note than in the $B u=O(1)$ regime, $R_{0} \approx L_{R}$ and the effects of stratification and rotation on these scales are balanced, but this does not necessarily imply a steady flow.

These classifications of flow regimes also correspond to different approximations of the equations of geophysical flows (see Charney 1948; Atkinson 1981; Cullen 2002). On synoptic scales where $R_{0}$ is much greater than $L_{R}$, the flows are quasi-geostrophic and hydrostatic, while on the meso-scale where $R_{0} \approx L_{R}$, the flows are non-geostrophic. In both cases they are hydrostatic if $R_{0} \gg H$. At the micro-scale where $R_{0} \ll L_{R}$ the flows are non-geostrophic. The dynamics of the front is non-hydrostatic and its structure is affected by turbulence (Westerweel et al. 2002). Classical linear geostrophic adjustment theory (e.g. Haltiner \& Williams 1980; Gill 1982) uses linearized shallowwater equations to calculate the end state of geostrophic balance given general initial data not satisfying the geostrophic relation. These results have been extended to nonlinear regimes of rotating shallow-water and Euler-Boussinesq (primitive) equations, extending both the general mathematical analyses of Babin, Mahalov \& Nicolaenko (1997a, c, 1998, 2002) and Embid \& Majda (1996), who obtained error bounds for solutions, and the study of weakly nonlinear geostrophic adjustment flows by Reznik, Zeitlin \& Jelloul (2001). The remarkable feature of the nonlinear terms (over the 'flat' areas of the current, excluding the frontal zones) is that they have a weak effect on the linear form and decay of the oscillation. A simple physical argument is proposed.

This paper is organized as follows. In $\S 2$, we consider perturbations for small $\mathrm{ft}$ from the von Kármán (1940) solution for a gravity current head in a weakly rotating flow, and then generalize these results to gravity currents on sloping surfaces, using the approach of Rottman et al. (1985). Two fluid systems are considered here. The analysis shows that on a horizontal surface, the slope of the front decreases and progressively vertical accelerations decrease implying that the vertical pressure gradient becomes hydrostatic. If the solution obtained with a perturbation analysis for $f t \ll 1$ is extrapolated, it leads to an estimate for the speed of the gravity current front and the time when the front stops. When the parameter $f t$ becomes of order one, there is a transition to another regime which is analysed with direct numerical simulations in $\S 2.3$. In $\S 2.2$, we analyse the impact of sloping surfaces on gravity currents. In $\S 2.3$, we compare our theoretical results for inviscid density currents over level surfaces with numerical solutions to Navier-Stokes equations (Pacheco \& Peck 2000; Pacheco 2001) which simulates a gravity current starting from rest and is particularly suitable for studying frontal dynamics. In $\S 3$, a numerical simulation is presented for the inviscid shallow-water code, that accurately models the final stages of the front where its slope is small and the hydrostatic approximation is valid (Pacheco \& Pacheco-Vega 2003). We also present detailed transition curves for initial conditions where $L_{R}<R_{0}$ lie between 0.33 and 0.66 . Rotation is applied after the gravity current is initiated, so that its effect can be seen more clearly. Significant nonlinear oscillations are observed. A summary of the results is presented in $\S 4$.

\section{Short time effects of weak rotation on gravity current fronts}

\subsection{Theoretical analysis on a horizontal surface}

\subsubsection{Formulation of the problem}

Using the notation of figure 2, we now state the equations for the velocity $\boldsymbol{U}$ and pressure $P$ fields within the current $[\mathrm{C}]$ and in the exterior region [E]. The variables 
(ii)

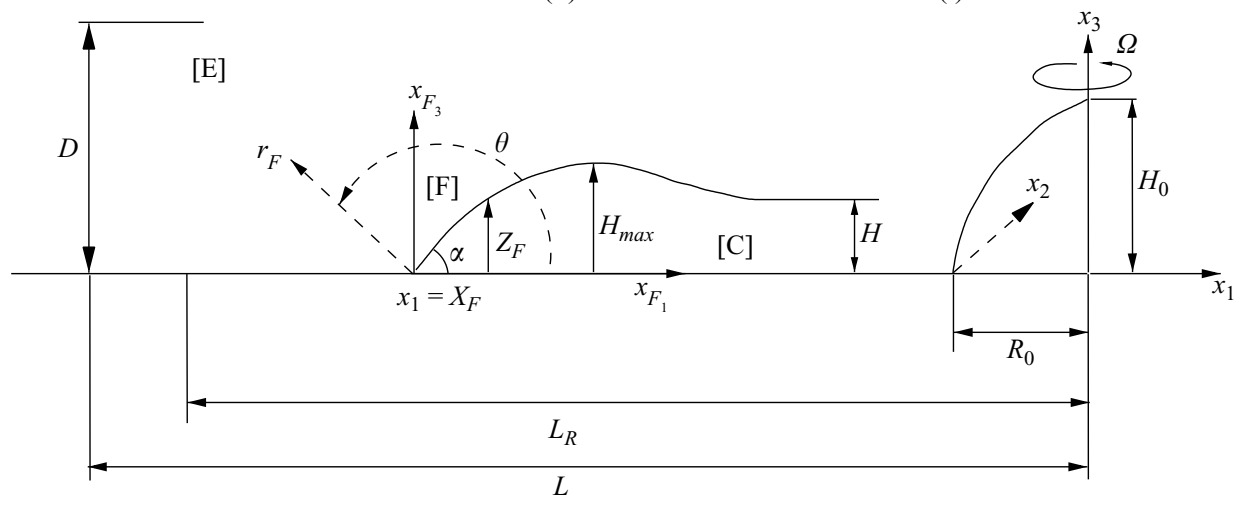

FigURE 2. Flow configuration for a gravity current that exists in the space of $0 \leqslant Z \leqslant Z_{F}(x, t)$. The front $[\mathrm{F}]$ of the density current is travelling at a velocity $-U_{F}$ in the absence of background rotation. $[\mathrm{C}]$ and $[\mathrm{E}]$ correspond to the interior and external flow regions and are indicated on the figure. (i) is initial $t=0$; (ii) current $f^{-1} \gg t>0$.

in each of these domains are identified by superscripts (C) and (E), respectively. It is assumed here, following Benjamin (1968), that near the front $[\mathrm{F}]$ the flow is inviscid and that no mixing takes place at the moving interface between the two domains, despite a discontinuity in tangential velocity across it. Thus we neglect the interfacial turbulent shear stresses, although in practice they contribute to the mean motion within $[\mathrm{C}]$ and the sharpness of the interface between $[\mathrm{C}]$ and $[\mathrm{E}]$ (Britter \& Simpson 1978; Westerweel et al. 2002). In a later section, we consider how body forces and the changing shape of the interface [I] between $[C]$ and $[E]$ induce a motion $\boldsymbol{U}^{(C)}$ within $[C]$. The density within $[C]$ is assumed to have a uniform value that is greater by $\Delta \rho$ than the density in [E] where the density is $\rho_{0}$. Note that $\Delta \rho$ is small compared to $\rho_{0}$, so that the Boussinesq approximation can be applied. Since the acceleration due to gravity $g$ is much greater than the vertical and horizontal acceleration of the fluid flow, the effect of this density variation is only to produce a buoyancy force $\boldsymbol{g} \Delta \rho / \rho_{0}=g^{\prime} \hat{\boldsymbol{x}}_{3}$ (unit vectors are denoted here by $\hat{\boldsymbol{x}}_{i}$ ). The pressure is normalized on $\rho_{0}$ (and the ambient hydrostatic pressure gradient $\left(-\boldsymbol{g} \rho_{0}\right)$ is subtracted out).

In the initial boundary-value problem, we solve the local solution near the front, which therefore can be applied to other shallow currents. It is assumed that, initially, for $t_{F}=\left(t-T_{R}\right)<0$, before the rotational Coriolis force is applied at $t=T_{R}$, the front of the density current at $x_{1}=+x_{F}$ is travelling steadily at a velocity $\left(-U_{F}\right)$. This enables the two-dimensional non-hydrostatic equations to be analysed in a coordinate frame $\left(x_{F_{1}}, x_{F_{3}}\right)$ moving at a velocity $-U_{F}$ for $t_{F}>0$.

We begin by considering a gravity current that exists in the space $0 \leqslant x_{F_{3}} \leqslant Z_{F}\left(x_{F}\right)$, where $Z_{F}=0$ for $x_{F_{1}}=x_{1}-X_{F}<0$ and $Z_{F}$ tends to a constant height $H$ far from the front (i.e. where $x_{F_{1}} / H \rightarrow \infty$ ). As we see later and in $\S 3.2$, when we analyse the overall development of a real current, $U_{F}$ and $H$ vary with time from their initial values of $U_{F_{0}}$ and $H_{0}$; but slowly on the time scale $H / U_{F}$. This situation corresponds to conditions on the left-hand side of figure 2. In $\S 2.1 .3$, we evaluate how the flow changes for $t>T_{R}$. Experimentally, this implies that initially, in the dense layer over a radius $R_{0}$, Coriolis forces are weak compared to the inertial forces, i.e. $L_{R} / R_{0} \gg 1$. The zero-order approximation in $\S 2.1 .2$ is valid when the time (in relation to $f^{-1}$ ) is small, i.e. $f t_{F} \ll 1$. The matching conditions across the interface, defined 
as $G_{F}=x_{F_{3}}-Z_{F}(x, t)=0$, are that the difference in normal velocity across it is zero and that the interface is a fluid surface so that its constituent fluid elements always remain within it.

The governing differential equation and boundary conditions can now be stated. In $[\mathrm{E}]$,

$$
\begin{gathered}
\frac{\mathrm{D} \boldsymbol{U}^{(E)}}{\mathrm{D} t}+f \hat{\boldsymbol{x}}_{3} \times \boldsymbol{U}^{(E)}=-\nabla P^{(E)}, \\
\boldsymbol{\nabla} \cdot \boldsymbol{U}^{(E)}=0,
\end{gathered}
$$

while in $[C]$,

$$
\begin{gathered}
\frac{\mathrm{D} \boldsymbol{U}^{(C)}}{\mathrm{D} t}+f \hat{\boldsymbol{x}}_{3} \times \boldsymbol{U}^{(C)}+g^{\prime} \hat{\boldsymbol{x}}_{3}=-\nabla P^{(C)}, \\
\nabla \cdot \boldsymbol{U}^{(C)}=0 .
\end{gathered}
$$

The kinematic boundary conditions applied at $x_{F_{3}}=0$, are

$$
\boldsymbol{U}^{(C)} \cdot \hat{\boldsymbol{x}}_{3}=\boldsymbol{U}^{(E)} \cdot \hat{\boldsymbol{x}}_{3}=0 .
$$

Far upstream of the current $\left(x_{F_{1}} \rightarrow-\infty\right)$,

$$
\begin{gathered}
\boldsymbol{U}^{(E)} \rightarrow U_{F} \hat{\boldsymbol{x}}_{1}, \quad P^{(E)}=\text { const. }=0, \\
x_{F_{1}} \rightarrow \infty\left(G_{F}>0\right), \quad \boldsymbol{U}^{(E)} \rightarrow U_{F} \hat{\boldsymbol{x}}_{1}, \quad P^{(E)}=0 .
\end{gathered}
$$

For $t_{F}<0$ when there is no rotation $(f=0)$ and $\mathrm{D} / \mathrm{D} t=0$, the far-field boundary condition in $[C]$ is then $\boldsymbol{U}^{(E)}=0$. With rotation, when $\mathrm{D} / \mathrm{D} t \neq 0$ and $t_{F}>0$, a boundary condition consistent with the exterior solution is derived in $\S$ 2.1.2.

Across the interface between regions $[\mathrm{C}]$ and $[\mathrm{E}]$ defined by $G_{F}=x_{F_{3}}-Z_{F}\left(x_{F_{1}}, t\right)=0$ the conditions are:

$$
\left(\boldsymbol{U}^{(E)}-\boldsymbol{U}^{(C)}\right) \cdot \nabla G_{F}=0, \quad P^{C}=P^{E},
$$

and

$$
\frac{\partial Z_{F}}{\partial t}+\frac{1}{2}\left(\boldsymbol{U}^{(E)}+\boldsymbol{U}^{(C)}\right) \cdot\left(\hat{\boldsymbol{x}}_{3}-\hat{\boldsymbol{x}}_{1} \frac{\partial Z_{F}}{\partial x_{F_{1}}}\right)=0 .
$$

The solution is obtained for small time $\left(f t_{F} \ll 1\right)$ using Maclaurin series expansions; so that

$$
\boldsymbol{U}^{(E)}(\boldsymbol{x}, t)=\boldsymbol{U}^{(E, 0)}\left(x_{F_{1}}, x_{F_{3}}\right)+\sum_{n=1}^{\infty}\left(f t_{F}\right)^{n} \boldsymbol{U}^{(E, n)}\left(x_{F_{1}}, x_{F_{3}}\right),
$$

and similarly for $\boldsymbol{U}^{(C)}, P^{(E)}$ and $P^{(C)}$. Continuity $(2.2 b)$ can be satisfied by expressing $\boldsymbol{U}$ in form of a streamfunction: $\Psi^{(E, n)}\left(x_{F_{1}}, x_{F_{3}}\right)$. Note that, when $f=0$, in the gravity current $\boldsymbol{U}^{(C, 0)}=0$ (Benjamin 1968).

The height $Z_{F}$ of the current also has an expansion

$$
Z_{F}\left(x_{F_{1}}, t_{F}\right)=Z_{F}^{(0)}\left(x_{F_{1}}\right)+\sum_{n=1}^{\infty}\left(f^{n} t_{F}^{n+1}\right) Z_{F}^{(n)}\left(x_{F_{1}}\right) .
$$

\subsubsection{Solution for zero order}

The zeroth order steady-state solution for the exterior region $[\mathrm{E}], \Psi^{(E, 0)}$, has a local (von Kármán) form for the inviscid and irrotational upstream flow, that is defined by the slope angle $\alpha$ of the front (where $Z_{F}=x_{F_{1}} \tan \alpha$ ), namely, of the front (where 
$\left.Z_{F}=x_{F_{1}} \tan \alpha\right)$, namely,

$$
\Psi^{(E, 0)}=A r_{F}^{\beta} \sin (\beta(\pi-\theta))
$$

where $r_{F}^{2}=x_{F_{1}}^{2}+x_{F_{3}}^{2}, \theta=\tan ^{-1}\left(x_{F_{3}} / x_{F_{1}}\right)$, where $A$ is a dimensional constant that defines the strength of the flow around the stagnation point and $\beta=1 /(1-\alpha / \pi)$. Thus, on $x_{F_{3}}=Z_{F}$ or $\theta=\alpha$,

$$
\left|U^{(E, 0)}\right|=\beta A r^{(\beta-1)}=\beta A\left(Z_{F} / \sin \alpha\right)^{(\beta-1)} .
$$

Matching the vertical hydrostatic pressure field in the front to the Bernoulli pressure gradients in the external flow, leads to

$$
-\frac{1}{2}\left|U^{(E, 0)}\left(x_{F_{1}}, Z_{F}\right)\right|^{2}=-g^{\prime} Z_{F} .
$$

Thence from (2.7),

$$
2(\beta-1)=1, \quad \text { whence } \quad \beta=3 / 2, \quad \alpha=\pi / 3 ;
$$

also

$$
A^{2}=g^{\prime}(4 \sqrt{3} / 9) \text {. }
$$

Note that the value of $A$ cannot be determined in terms of $U_{F}$ by this calculation, since its dimensions (when $\beta=3 / 2$ ) are $L^{1 / 2} T^{-1}$.

However (for reasons given by Benjamin 1968), downstream of the front where the height of the current $Z_{F}$ reaches a constant value $H, \boldsymbol{U}^{(E, 0)}$ approaches its upstream value $U_{F} \hat{\boldsymbol{x}}_{1}$, and $P^{(E, 0)}=0$. It follows from $(2.2 a)$ that

$$
0=\frac{1}{2 U_{F}^{2}}-H g^{\prime}, \quad \text { whence } \quad H=\frac{1}{2}\left(\frac{U_{F}^{2}}{g^{\prime}}\right) .
$$

The transition between the von Kármán stagnation point flow and this downstream flow requires the surface of the current to have a maximum height $H_{\max }$ where $x_{F_{1}}=x_{\max }$. There has to be some dissipation in the flow between $x_{F_{1}}=x_{\max }$ and $x \rightarrow \infty$. Approximate theory for this region and experiments show that $H_{\max } \approx 1.2 H$ (Britter \& Simpson 1978) and $A \approx U_{F} / H^{1 / 2}$.

To estimate the flow far upwind and above the gravity current, it can be assumed that the potential flow in [E] over the current is similar to that over a hill or an airfoil with a low slope (e.g. Milne-Thompson 1968). Therefore, when $\sqrt{x_{1}^{2}+x_{3}^{2}} / H \geqslant 1$,

$$
\Psi^{(E, 0)} \approx-U_{F} H \tan ^{-1}\left(\frac{x_{F_{3}}}{x_{F_{1}}}\right)+U_{F} x_{F_{3}} .
$$

\subsubsection{Leading-order effects of weak rotation}

To calculate the terms of order $f t_{F}$ and $\left(f t_{F}\right)^{2}$ in the expansion (2.6), we assume that it is a regular expansion, which implies that the shape $\left(x_{F_{3}}=Z_{F}\left(x_{F_{1}}\right)\right)$ of the front is unchanged to first order. We denote these terms by $\tilde{U}^{(E, 1)}, \tilde{U}^{(E, 2)}$. Then we consider whether it is necessary to relax this constraint, to calculate the complete terms at these orders (as in the calculation of the shear effect by Rottman et al. 1985).

As usual in geophysical analysis, take the $x_{F_{1}}$ coordinate to the east, and $x_{F_{2}}$ coordinate to the north. We develop a solution based on the assumption that the front remains straight and parallel to the $x_{F_{2}}$-axis. Therefore all gradients parallel to the $x_{F_{2}}$ direction are assumed to be zero (this is a close approximation to the flow near the front of an axisymmetric current, analysed in $\$ 2.3$ ). Because a moving coordinate system is used in a fluid with an overall rotation, this effectively introduces a spanwise 
or north-south pressure gradient $\partial P_{\infty} / \partial x_{F_{2}}=-f U_{F}$. Thus, to first order, (2.1) becomes

$$
\tilde{U}_{2}^{(E, 1)}+U_{1}^{(E, 0)}=U_{F}
$$

and

$$
\tilde{U}_{1}^{(E, 1)}+U_{3}^{(E, 0)}=0
$$

thence $P^{(E, 1)}=0$. Thus, since there is no motion perpendicular to it, the rate of change of the frontal surface is unchanged to this order, so that $Z_{F}^{(1)}=Z_{F}^{(2)}=0$. Hereinafter, the tilde superscripts for the second-order terms can be dropped. There are significant effects in the current $[\mathrm{C}]$ caused by the pressure gradient. Note that from $(2.13 a)$ and (2.4a) $U_{2}^{(E, 1)} \rightarrow 0$ as $x \rightarrow-\infty$. To first order,

$$
U_{1}^{(C, 1)}=0, \quad U_{2}^{(C, 1)}=-U_{F_{0}}, \quad U_{3}^{(C, 1)}=0 .
$$

Note that the initial effect of rotation is to induce anticyclonic motion $U_{2}(>0)$ not only near the front in $[\mathrm{E}]$, but everywhere inside the current [C] (see also the Appendix A). The motions in $[\mathrm{E}]$ are given (to second order) by:

$$
\begin{gathered}
2 U_{1}^{(E, 2)}-U_{2}^{(E, 1)}=-\frac{\partial P^{(E, 2)}}{\partial x_{F_{1}}}, \\
2 U_{3}^{(E, 2)}=-\frac{\partial P^{(E, 2)}}{\partial x_{F_{3}}}, \\
2 U_{2}^{(E, 2)}+U_{1}^{(E, 0)} \frac{\partial U_{2}^{(E, 1)}}{\partial x_{F_{1}}}+U_{3}^{(E, 1)} \frac{\partial U_{3}^{(E, 1)}}{\partial x_{F_{3}}}+U_{1}^{(E, 1)}=0,
\end{gathered}
$$

and to third order in $[\mathrm{C}]$,

$$
\begin{aligned}
& 2 U_{2}^{(C, 2)}+U_{1}^{(C, 1)}=0, \\
& 3 U_{2}^{(C, 3)}+U_{1}^{(C, 2)}=0 .
\end{aligned}
$$

Therefore, from $(2.13 b), U_{2}^{(E, 2)}=0$. Using the continuity equation (and the fact that $\partial U_{2} / \partial x_{F_{2}}=0$ to all orders), we can express $\boldsymbol{U}^{(E, 2)}$ in terms of the second-order streamfunction $\Psi^{(E, 2)}$, namely,

$$
U_{1}^{(E, 2)}=\frac{\partial \Psi^{(E, 2)}}{\partial x_{F_{3}}}, \quad U_{3}^{(E, 2)}=-\frac{\partial \Psi^{(E, 2)}}{\partial x_{F_{1}}} .
$$

Taking the curl of (2.14), using (2.13), leads to an inhomogeneous equation for $\Psi^{(E, 2)}$

$$
\left(\frac{\partial^{2}}{\partial x_{1}^{2}}+\frac{\partial^{2}}{\partial x_{3}^{2}}\right) \Psi^{(E, 2)}+\frac{1}{2} \frac{\partial^{2}}{\partial x_{3}^{2}} \Psi^{(E, 0)}=0 .
$$

Following the assumption set out at the beginning of $\S 2.1 .3$, the boundary between $[\mathrm{C}]$ and $[\mathrm{E}]$ is not changed, to order $\left(f t_{F}\right)^{2}$. Therefore, $\Psi^{(E, 2)}$ satisfies $(2.15 b)$ in the same domain as $\Psi^{(E, 0)}$ (i.e. outside the current whose shape is still the same). Its boundary/matching conditions are set out in (2.3) and (2.5) where

$$
\left.\begin{array}{l}
\Psi^{(E, 2)}=0 \quad \text { on } \quad x_{F_{3}}=0 \quad \text { for } \quad x_{F_{1}}<0, \\
\Psi^{(E, 2)}=\Psi^{(C, 2)} \quad \text { on } \quad x_{F_{3}}=Z_{F}^{(0)}=x_{F_{1}} \tan \alpha \quad \text { for } x>0 .
\end{array}\right\}
$$

Here, $\alpha=\pi / 3$ and $\Psi^{(C, 2)}$ is the streamfunction for the perturbation flow with the current $[\mathrm{C}]$. Equation (2.15b) near the stagnation point, as $r_{F} \rightarrow 0$, using (2.7) and 
(2.9), reduces to

$$
\nabla^{2} \Psi^{(E, 2)}=\frac{3}{4} A r_{F}^{-1 / 2} \sin \left(\frac{1}{2}(\pi-\theta)\right)
$$

and, as $(r / H) \rightarrow \infty$, to

$$
\nabla^{2} \Psi^{(E, 2)}=\frac{q^{(0)}}{r_{F}^{2}} \sin (2(\pi-\theta))
$$

where $q^{(0)} \approx U_{F_{0}} H$ is the effective source strength of the far-field perturbation for the zero-order solution in (2.12). Therefore, the solutions as $r / H \rightarrow 0$, are

$$
\Psi^{(E, 2)}=\beta_{F} r_{F}^{3 / 2} \sin \left(\frac{3}{2}(\pi-\theta)\right)+\frac{3}{8}\left(A r_{F}^{3 / 2} \sin \left(\frac{1}{2}(\pi-\theta)\right)\right)+\frac{1}{2} \frac{U_{F} x_{F_{3}}}{2},
$$

where $\beta$ is an unknown coefficient for the complementary function. As $r_{F} / H \rightarrow \infty$,

$$
\Psi^{(E, 2)}=-\frac{1}{4} q^{(0)} \sin (2(\pi-\theta))+q^{(2)}(\pi-\theta) .
$$

Since the complementary function is zero on the boundary of [E], its arbitrary coefficient $\beta$ can be shown to be zero when the pressure fields are balanced. Note the term $U_{F} x_{F_{3}} / 2$ in the near field is the Coriolis induced flow that enters into the current (which effectively slows it down); but since $\boldsymbol{U}^{(E, 2)} \rightarrow 0$ as $\left(r_{F} / H\right) \rightarrow \infty$, there must be a balancing far-field source solution, such that $q^{(2)} \simeq U_{F}(H / 2)$.

Now, by applying the boundary conditions (2.5), the second-order solution for $\Psi^{(C, 2)}$ inside the current can be derived. Since there is no zeroth-order motion in [C], it follows that

$$
\nabla^{2} \Psi^{(C, 2)}=0 .
$$

The second-order velocities normal to the surface $G_{F}$ must match each other on $x_{F_{3}}=Z_{F}^{(0)}(x)$ and satisfy $(2.3)$ on $x_{F_{3}}=0$.

The conditions far from the front can be derived from (2.2a). Since these are constant in [E] and [C], it follows that $\partial P^{(C, 2)} / \partial x_{F_{1}}=0$ and therefore from $(2.13 c)$ as $x_{F_{1}} \rightarrow \infty, \Psi^{(C, 2)}=\left(U_{F} / 2\right) x_{F_{3}}$. Thus, as $r_{F} / H \rightarrow 0$,

$$
\Psi^{(C, 2)}=\frac{3}{16} \sqrt{3} A r_{F}^{3 / 2} \sin \left(\frac{3}{2} \theta\right)+\frac{1}{2} U_{F} x_{F_{3}} .
$$

Hence, the second-order term for the horizontal perturbation velocity in the current (on the ground at $x_{F_{3}}=0$ ) at a radius $r_{F}$ from the front $[\mathrm{F}]$ is

$$
U_{1}^{(C, 2)}=\frac{9}{32} \sqrt{3} A r_{F}^{1 / 2}+\frac{1}{2} U_{F_{0}} .
$$

Thence, in fixed coordinates (denoted with an asterisk) the total horizontal velocity on the ground in the current is

$$
U_{1}^{(C)}=-U_{F_{0}}\left(1-\frac{1}{2}\left(f t_{F}\right)^{2}\right)+\frac{9}{32} \sqrt{3} A r_{F}^{1 / 2}\left(f t_{F}\right)^{2} .
$$

Typically, this solution is valid for $r_{F} \lesssim H$. Thus, since $A \simeq U_{F} / H^{1 / 2}$, the velocity decreases from the front, where $r_{F}=0$, to its minimum value where $r_{F} \lesssim H$, given by

$$
U_{1(\min )}^{(C)} \simeq U_{F_{0}}\left\{-1+\left(f t_{F}\right)^{2}\left(\frac{1}{2}+\frac{9}{32} \sqrt{3}\right)\right\} .
$$

Note that the velocity at the leading edge of the front (where $r_{F}=0$ ) slows down less than at the point of greatest thickness. In other words, the front is moving faster than the fluid in the bulk of the current which is why the slope decreases with time.

If the solution (2.20) for $r_{F}=0$ is extrapolated, it leads to an approximate estimate for the speed of the front and the time when the front stops (taken from when rotation 
was initiated and the front was in a fully developed state), namely

$$
U_{F}=-U_{F_{0}}\left(1-\left(f t_{F}\right)^{2} / 2\right), \quad \text { so that } U_{F}=0 \text { when } f t_{F} \simeq \sqrt{2} .
$$

It follows from (2.19) and (2.14e) that

$$
U_{2}=-U_{F_{0}} f t_{F}-\left(\left(\frac{9}{32} \sqrt{3}\right) A r_{F}^{1 / 2}+\frac{1}{2} U_{F_{0}}\right) \frac{1}{3}\left(f t_{F} t\right)^{3},
$$

which shows that the transverse (anticylonic motion) increases with time and increases along the current from the front to a maximum value where $r_{F} \sim H / \alpha$. Therefore, at $t_{F}=\sqrt{2} / f$, the transverse velocity at $r_{F}=0$ is

$$
U_{2} \simeq-1.9 U_{F_{0}},
$$

and at $r_{F} \sim H / \alpha($ where $\alpha \simeq 0.1)$,

$$
U_{2} \simeq-3 U_{F_{0}} \text {. }
$$

Of course, this extrapolation is only indicative. At greater time, when the parameter $\mathrm{ft}_{F}$ becomes of order one, there is a transition to another regime which is considered in $\S \S 2.3$ and 3.2 .

The changes to the frontal surface in a fixed rotating frame can be derived (to order $\left.\left(f t_{F}\right)^{3}\right)$ by considering the azimuthal velocity at the front, from (2.18),

$$
Z_{F}^{*}=\left(x^{*}-U_{F_{0}} t+\frac{U_{F_{0}}}{2} \frac{f^{2} t_{F}^{3}}{8}\right) \tan \alpha,
$$

where $\alpha=(\pi / 3)-(3 \sqrt{3} / 32) A r_{F}^{1 / 2} f^{2} t_{F}^{3}$.

This shows how the slope decreases very rapidly at the front (in fact, $\mathrm{d} Z_{F} / \mathrm{d} r_{F}$ is singular as $r_{F} \rightarrow 0$ ). The change of shape of the current when terms of order $t_{F}^{3}$ are considered, is given by

$$
Z_{F}=x_{F_{1}} \tan \alpha-\cos \alpha \int_{0}^{t_{F}} \frac{\partial \Psi^{(C, 2)}}{\partial r_{F}}(f \tau)^{2} \mathrm{~d} \tau=\sqrt{3} x_{F_{1}}-\frac{3}{64} \sqrt{3} A r_{F}^{1 / 2} f^{2} t_{F}^{3},
$$

where $x_{F_{1}}=x_{1}^{*}-U_{F_{0}} t+U_{F_{0}} f^{2} t_{F}^{3} / 16$.

For very wide density currents (with scale $L \gg L_{R}$ ), the movement of the front does not affect the height $H$ (or the density) in the density current. In that case, its average slope $\alpha$ over the front of the current $\alpha_{C}$ is determined by the front dynamics, which are essentially two dimensional. It follows that in the geostrophic limit when $f t_{F} \geqslant 1$, the average slope is

$$
\alpha_{C} \sim \frac{H}{\int_{0}^{\sqrt{2} / f} U_{F} \mathrm{~d} t} \simeq \frac{H}{U_{F_{0}} / f} \simeq \frac{f}{\sqrt{g^{\prime} / H}}=f / N,
$$

where $N$ is the frequency of long-wavelength oscillations on the density current. Note that in this limit the transverse velocity $U_{2}$ estimated by (2.24) is of the same order as (but larger than) Margules' (1906) value derived from (2.27) assuming a sloping front and geostrophic equilibrium, namely $\alpha_{C} g^{\prime} / f=\sqrt{g^{\prime} H}$. Thus, at least for very wide currents, the small time expansion indicates a monotonic trend to the geostrophic equilibrium solution. Because the value of $U_{2}$ outside the density current obtained from this linear theory (see figure 2) exceeds the asymptotic value when $f t_{F} \sim 1$, it produces an excess pressure gradient in the $x_{1}$-direction. This is why the linear theory underpredicts (by $O\left(f^{-1}\right)$ ) the time to slow down the current. For a physical discussion of axisymmetric currents see Appendix A.1. 


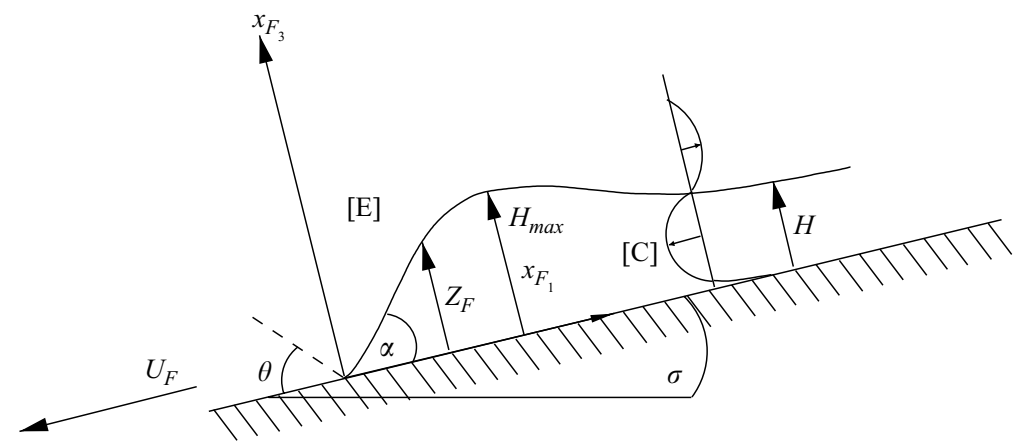

FIGURE 3. Density current on a sloping surface.

\subsection{Fronts of density currents on sloping surfaces}

\subsubsection{Motivation and background}

In many geophysical and environmental flows, density currents are found on sloping surfaces, the most well known being the current of snow particles in an avalanche and the slope katabatic flows occurring at night in mountainous areas. Another example is the katabatic flow along the slopes of the Antarctic plateau which extend over $1000 \mathrm{~km}$ and have vertical scales of order $1 \mathrm{~km}$ (Chen, Boyer \& Tao 1993). In the ocean, flows of order $10^{-1} \mathrm{~m} \mathrm{~s}^{-1}$ occur down slopes with gradients $(\sigma)$ of $10^{-2}$ to $10^{-1}$ over distances, of order of $10^{2} \mathrm{~km}$, that are much greater than the Rossby deformation radius $N H / f$ of $10 \mathrm{~km}$. In particular, gravity currents originating owing to river water discharging into coastal oceans extend tens of kilometres seawards (Legeckis \& Creswell 1981; Creswell \& Golding 1986), and it is noteworthy that these gravity currents are associated with large-amplitude unstable waves and eddies having horizontal lengths of order $50-100 \mathrm{~km}$ implying a strong influence of nonlinear dynamics in the adjustment process (Qiu \& Imasato 1988). Most previous studies involving slope flows and the front temperature have been experimental (e.g. Britter \& Linden 1980 , hereinafter referred to as B\&L). Many theories for steady currents have neglected the influence, if any, of fronts (e.g. Turner 1973, but see B\&L).

The purpose of this brief section is to point out, for completeness, the inviscid solution for steady gravity current fronts and geostrophic fronts on slopes and how these flows relate to those behind the front. As with currents on horizontal planes, the inviscid gravity current horizontal analysis gives physical insights and useful estimates for density currents.

\subsubsection{Inviscid solution for gravity current on a sloping surface}

Consider a current with density $\rho_{E}+\Delta \rho$ proceeding steadily down (or up) a slope (of $\sigma$ ) with velocity $U_{F}$ (see figure 3 for flow configuration). Unlike the case of a current on a horizontal plane, the local steadiness of currents mostly depends on the existence of a balance between those stresses that decelerate the flow caused by the shear stress gradient $\left(\partial \tau / \partial x_{F_{3}}\right)$ and inertial forces $\left(L H^{-1} \partial\left(U^{2} H\right) / \partial x\right)$ and the accelerating buoyancy forces acting down the slope $\Delta \rho g \sin \sigma$. As explained by Turner (1973) and demonstrated for the case of atmospheric gravity currents by Manins \& Sawford (1979), the latter of these two effects, the inertial force associated with entrainment, is the dominant decelerating force. This balance behind the front is necessary for fluids where the density in the current is a significant fraction of that in the external flow in [E]. However, where the density of the fluid in the gravity 
current is negligible (e.g. bubbles, where $(\rho+\Delta \rho / \rho) \ll 1)$ the flow is steady because of a balance of inviscid forces in the external region [E]. In either case, if the flow reaches a steady state, then an inviscid analysis is the leading-order approximation near the front because here inertial and buoyancy forces are large relative to the Reynolds stresses. This implies, as in $\S 2.1$, that in the frame of reference of the front, $\boldsymbol{U}^{(C)}=0$ and that $\boldsymbol{U}^{(E)}=0$ at the stagnation point.

In order to balance the linear variation of hydrostatic pressure in [C] with the pressure drop produced by acceleration along the interface, it is necessary as before that $\alpha=\pi / 3$. However, the constant $A$ in (2.10) which is increased slightly, is now given by

$$
A^{2}=g^{\prime} \frac{4}{9} 2 \sin \left(\frac{1}{3} \pi+\sigma\right)
$$

This formula can be used (as on a level plane) to estimate $U_{F}$, since $A^{2} \simeq U_{F}^{2} / H$. Thus,

$$
U_{F} \approx \sqrt{g^{\prime} H} \frac{2}{3} \sqrt{2} \sin ^{1 / 2}\left(\frac{1}{3} \pi+\sigma\right) .
$$

The result of (2.28) shows that $A^{2}$ is a maximum when $\sigma=\pi / 6\left(=30^{\circ}\right)$, when it is $(2 / \sqrt{3})$ times its value at $\sigma=0$ and a minimum when $\sigma=\pi / 2$, when $A^{2}$ is $1 / \sqrt{3}$ times its value at $\sigma=0$. Laboratory observations of Noh \& Fernando (1992) for a volume release are consistent with the results that $\alpha=\pi / 3$ on a slope. The implication of (2.29) is that for $0<\sigma<\pi / 3, U_{F}$ (for given $H$ ) increases by less than about $7 \%$ and for $0<\sigma<\pi / 2$ the variation is less than $30 \%$. B\&L find an even smaller variation (for $\pi / 30 \lesssim \sigma<\pi / 2$ ), but they do find that the maximum in $U_{F}$ occurs when $\sigma=\pi / 6$. The result (2.29) agrees well with the measurements of long bubbles in a sloping tube by Zukoski (1966) which is a physical situation closer to the idealized model.

\subsubsection{Geostrophic fronts on a slope}

The effect of rotation on density currents over a plane with slope $\sigma$, over a period of order $f^{-1}$ is also to bring the gravity current to rest and to set up an anticyclonic motion within [C] (e.g. Lentz \& Helfrich 2002). We consider the steady-flow solution at the front controlled by the Coriolis and hydrostatic forces.

As in $\S 2.1 .2$, in the asymptotic state where $f t_{F} \gg 1$, the geostrophic gradient in the front parallel to the surface is

$$
\frac{\partial P}{\partial x_{F_{1}}}=f \cos \sigma U^{(C)}-g^{\prime} \sin \sigma
$$

while the normal pressure gradient is hydrostatically determined, so that

$$
\frac{\partial P}{\partial x_{F_{3}}}=-g^{\prime} \cos \sigma
$$

The boundary condition is that $P=P_{s}=$ constant on $Z_{F}=x_{F} \tan (\alpha)$. It follows that the horizontal velocity in the current parallel to the front is given by

$$
U_{2}^{(C)}(\sigma)=g^{\prime} \frac{1}{f}(\tan \alpha+\tan \sigma)
$$

Therefore, the ratio of this cross-front velocity on a slope $\sigma$ to its value on a horizontal plane is

$$
\frac{U_{2}^{(C)}(\sigma)}{U_{2}^{(C)}(\sigma=0)}=\frac{\tan (\alpha)+\tan (\sigma)}{\tan (\alpha)}
$$


Since for a front in geostrophic equilibrium on a horizontal level plane

$$
\alpha \simeq \frac{f}{\left(g^{\prime} / H\right)^{1 / 2}} \simeq\left(\frac{f}{N}\right) \sim 10^{-2},
$$

and since from the analysis of $\S 2$, the value of $\alpha$ is not changed by a small slope, the solution (2.32) shows that, even on slopes where $\sigma>10^{-2}$, anticyclonic velocities generated near the front are significantly larger than on a flat surface. Therefore, large turbulent stresses and mixing would be initiated which would control this motion as the laboratory experiments (simulating Antarctic flows) of Chen et al. (1993) demonstrate. Another prediction of this analysis is that, since from (2.28), $A$ increases with bottom slope $(\sigma)$, it follows from $(2.24 a)$ that the slope of the front relative to the surface decreases more rapidly with time on a slope.

Comparison with numerical simulations of fronts on a slope without and with rotation are made in $\S 2.3$.

\subsection{Eulerian numeriacal simulation}

Time-dependent non-hydrostatic calculations of buoyancy-driven currents can be compared with the above inviscid solutions, where the two-phase flow motion of the gravity current is analysed in a two-dimensional domain. The gravity current is released along a floor of a tank of height $D$ and length $L$ containing a homogeneous fluid of density $\rho_{0}$, as shown in figure 2 . The initial volume of heavy fluid, of density $\rho$, has the shape of a right parallelepiped of height $H_{0}$ and length $R_{0}$. The equations governing the flow in the Boussinesq limit, written in the primitive variable formulation are:

$$
\begin{aligned}
\frac{\partial U_{j}}{\partial x_{j}} & =0 \\
\frac{\partial U_{i}}{\partial t}+\frac{\partial}{\partial x_{j}}\left(U_{j} U_{i}\right) & =-\frac{1}{\rho_{0}} \frac{\partial p}{\partial x_{i}}-\frac{\rho-\rho_{0}}{\rho_{0}} g_{i}-\epsilon_{i j k} f_{j} U_{k}, \\
\frac{\partial \rho}{\partial t}+\frac{\partial}{\partial x_{j}}\left(\rho U_{i}\right) & =\kappa \frac{\partial^{2} \rho}{\partial x_{j} \partial x_{j}},
\end{aligned}
$$

where $i, j=1,3 ; U_{i}$ represents the Cartesian velocity components; $p$ is the pressure; $\rho, \Delta \rho$ and $\rho_{0}$ represent density, density difference and reference density; $\kappa$ is the coefficient of diffusion for density; $g_{i}$ the gravity, and $f_{j}$ the Coriolis parameter. (Note $f_{j}$ is twice the angular velocity and is applied at time $T_{R}$ ). The boundary conditions at $t=0$ are

$$
\begin{aligned}
U_{i} & =0 \quad\left(0 \leqslant x_{1} \leqslant L, 0 \leqslant x_{3} \leqslant D\right), \\
\rho & = \begin{cases}\rho_{0}+\Delta \rho & \left(0 \leqslant x_{1} \leqslant R_{0}, 0 \leqslant x_{3} \leqslant H_{0}\right), \\
\rho_{0} & \text { elsewhere. }\end{cases}
\end{aligned}
$$

The boundary conditions for $t \geqslant 0$ on all boundaries are

$$
\begin{aligned}
U_{i} n_{i} & =0, \\
n_{i} \frac{\partial U_{j}}{\partial x_{i}} & =0, \\
n_{i} \frac{\partial \rho}{\partial x_{i}} & =0 .
\end{aligned}
$$

The non-staggered-grid layout is employed in this analysis. The pressure and the Cartesian velocity components are defined at the cell centre and the volume fluxes 
are defined at the midpoint of their corresponding faces of the control volume in the computational space. We use a semi-implicit time-advancement scheme with the Adams-Bashforth method for the explicit terms and the Crank-Nicolson method for the implicit terms as described by Zang, Street \& Koseff (1994). In this method, the density variation is computed by coupling the transport equation for density with the momentum equation in a Eulerian frame. Slip boundary conditions were specified along the walls. The simulations were initialized with the fluid at rest. Note that the diffusivity $\kappa$ is non-zero for numerical stability, but its value has a negligible effect on the results.

As the gravity current is released from the rest, a mutual intrusion flow develops from an initial state as the gravity current evolves in time. After a brief acceleration phase, the front reaches a constant velocity. Then, the effect of rotation is initiated (at $t=T_{R}$ ) causing a decrease of the front velocity in the $x_{1}$-direction. After some time, the front stops and a sequence of oscillations follow.

In order to avoid the effects of the upper boundary, the initial height $H_{0}$ was $1 / 5$ of the depth $D$. For the simulations reported here $D=5 \mathrm{~m}, L=10 \mathrm{~m}, R_{0}=2 \mathrm{~m}$, $g=10 \mathrm{~m} \mathrm{~s}^{-2}, \rho_{0}=1.0 \mathrm{~kg} \mathrm{~m}^{-3}$ and $\Delta \rho=0.1 \mathrm{~kg} \mathrm{~m}^{-3}$. The computational mesh had $(1000 \times 500)$ grid points for a value of molecular diffusivity $\kappa=2.5 \times 10^{-4} \mathrm{~m}^{2} \mathrm{~s}^{-1}$. The position of the front was assumed to be at the point where the local density at the bottom (sweeping from right to left) reached a value of $98 \%$ of the gravity current density $\left(1.08 \mathrm{~kg} \mathrm{~m}^{-3}\right)$. The value of the Coriolis parameter was set to $f=1.5 \mathrm{~s}^{-1}$ and the time at which rotation was initiated was set to $T_{R}=4.5 f^{-1}$.

The position and velocity of the front for $H_{0} / D=1 / 5$, so that $L_{R} / R_{0}=0.33$ are shown in figure 4 as function of dimensionless time $f t_{F}$ (bottom horizontal axis) with and without rotation for slope values of $\sigma=0, \pi / 12$. The Coriolis parameter $f=1.5 \mathrm{~s}^{-1}$ was used in the normalization of time (for both the rotating and nonrotating cases) so that the plots can be on the same graph. A comparison of the numerical results with the perturbation theory in the figure, displays the time for which the gravity current propagation is inhibited by rotation. Also it is shown that with rotation, the gravity current oscillates after the front reaches the Rossby radius of deformation instead of stopping completely. Figure 4 demonstrate that without rotation, the front reaches a constant velocity with and without slope; but the velocity of the front is higher on the sloping surface by about $20 \%$, which is larger than the theoretical estimate of $\S 2.2$. Note that if the front speed $U_{F}$ is normalized on the average height $H(t)\left(\simeq 0.3 H_{0}\right)$, it is found that the local ratio $U_{F} /\left(g^{\prime} H\right)^{1 / 2} \simeq 1.0$, which is close to the value found in many two-dimensional and three-dimensional experiments. On a slope of $\sigma=\pi / 2$, the local ratio is about 1.2 , which is higher than for the laboratory experiments (where surface stress is more important) (B\&L). With rotation, the gravity current front first halts (and reverses) at $f t_{F} \approx 2.25$ with and without slope, showing that the slope has a weak effect on the time required for the front to stop. The dashed line in figure 4 indicates the time where the rotation is imposed $\left(t=T_{R}\right)$ upon the gravity current has reached constant velocity. The perturbation theory, described in $\S 2.1 .3$, leads to an estimated time when the rotating gravity current halts $f t_{F}=\sqrt{2}$. The dash-dot line in the same figure shows that the theoretical extrapolation for $t_{F}$ is an underestimate by about $50 \%$. Slope results in figure 4 are consistent with the theory of $\S 2.2 .3$ that the current even halts on a slope (which is not unexpected). Note that the time at which the front halts is approximately the same with and without the slope. The Eulerian simulations show persistence of vortex structures owing to the absence of three-dimensional breakdown, thence, baroclinic instability is being suppressed. 

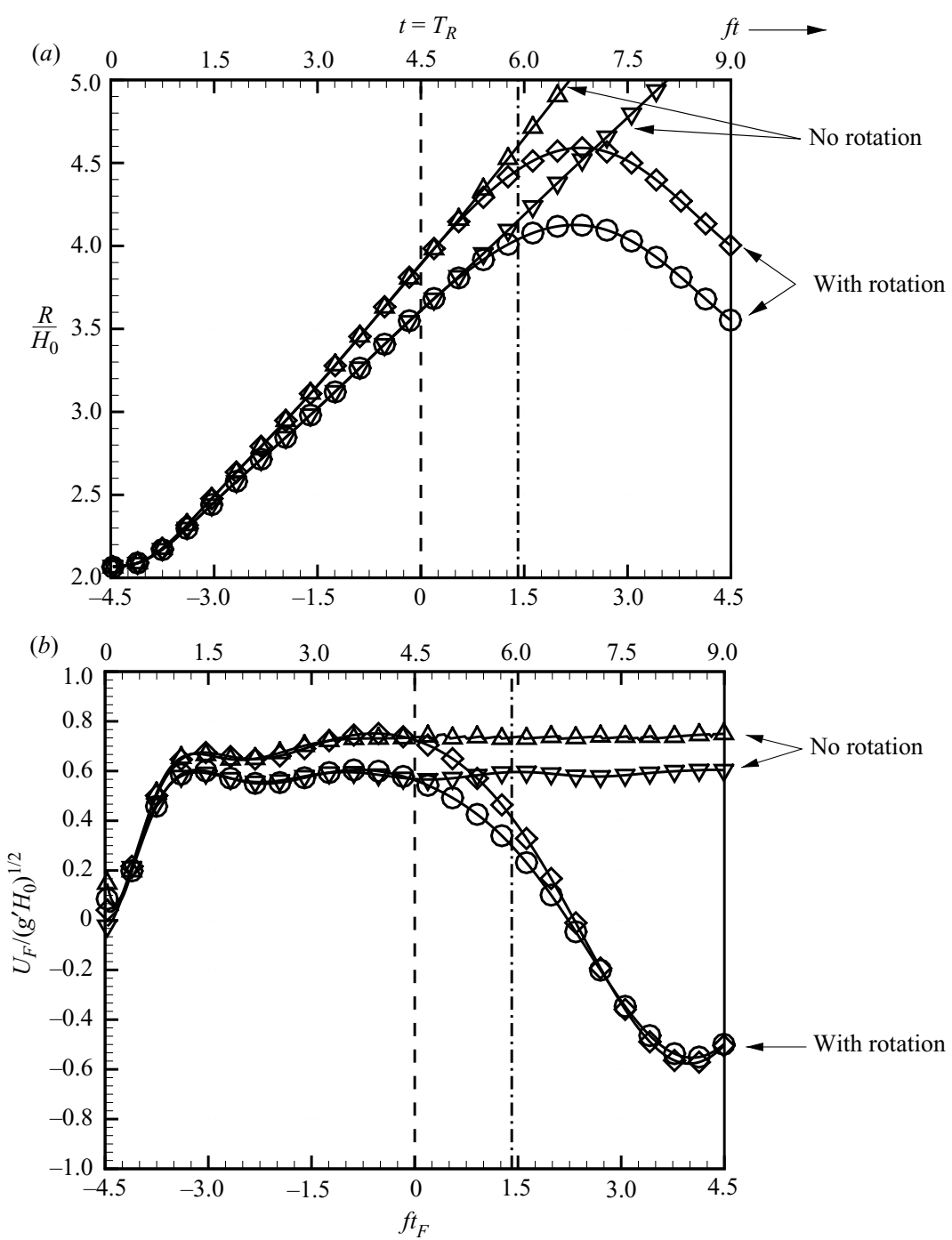

Figure 4. (a) Position of the front. (b) Velocity of the front. Numerical simulations of the twodimensional density current using non-hydrostatic (inviscid) Eulerian equations for $H_{0} / D=1 / 5$ as a function of dimensionless time and slope $\sigma$. Note that rotation is initiated at $t=T_{R}$ and $t-T_{R}=t_{F} . \nabla, f=0 \mathrm{~s}^{-1}$ and $\sigma=0 ; \Delta, f=0 \mathrm{~s}^{-1}$ and $\sigma=\pi / 12 ; O, f=1.5 \mathrm{~s}^{-1}$ and $\sigma=0 ; \diamond$, $f=1.5 \mathrm{~s}^{-1}$ and $\sigma=\pi / 12$. $-\cdot-$ the time when the velocity of the front decreases to zero. The timeline is an estimate based on the perturbation theory.

Density contour plots at the dimensionless time $f t_{F}=2.25$, are shown in figure 5 . For these simulations only the initial current height $H_{0}$ and half of the length of the channel are plotted. Figure 5(a) corresponds to the case where the slope is zero and figure $5(b)$ depicts the gravity current density for a slope $\sigma=\pi / 12$. Note how on the slope the 'head' of the current is higher and the centre is more depressed.

Note how the contours of the transverse velocity $U_{2}$, plotted in figure 6 for the same time, extend into the external flow, as shown in the linear theory. The dashed lines in the figure indicate regions where the transverse velocity is positive and the solid lines, regions of negative transverse velocity. The maximum value for the velocity $U_{2}$ 

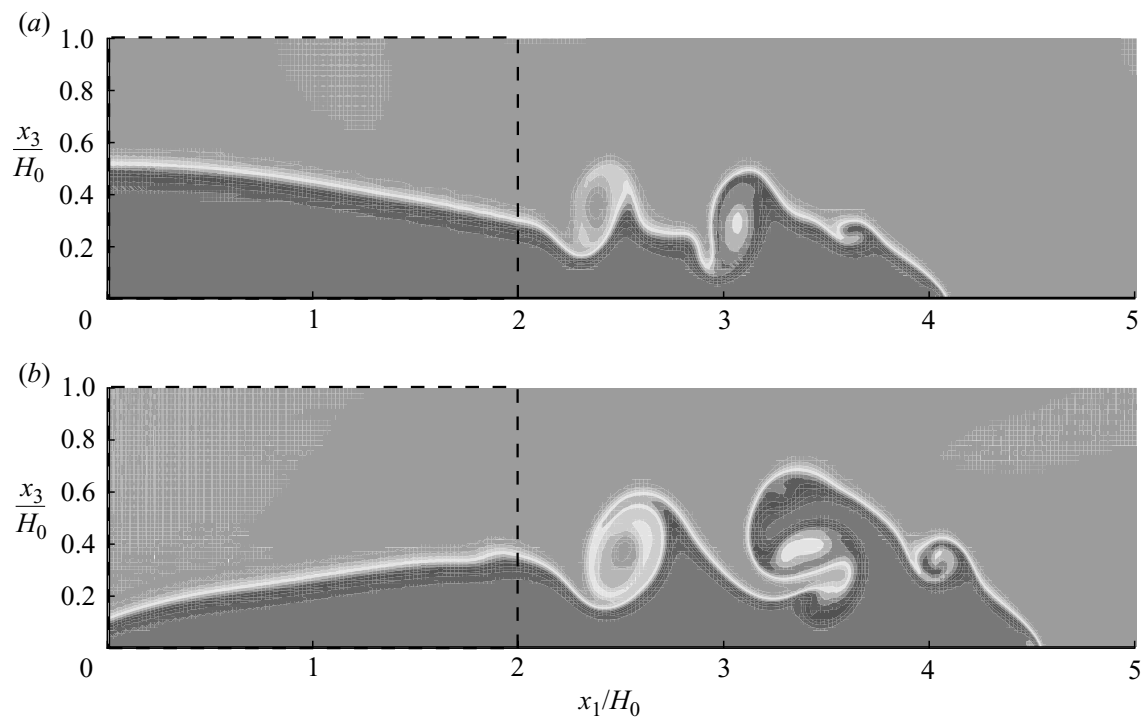

Figure 5. Non-hydrostatic Eulerian numerical simulations for two-dimensional gravity currents with rotation (initiated at time $T_{R}=4.5 f^{-1}$ ). Density fields for $H_{0} / D=1 / 5, f t_{F}=2.25$, $H_{0} / R_{0}=1 / 2, L_{R} / R_{0}=0.33$. (a) slope $\sigma=0,(b) \pi / 2$. The dashed line indicates the location of the gravity current at $t=0$.

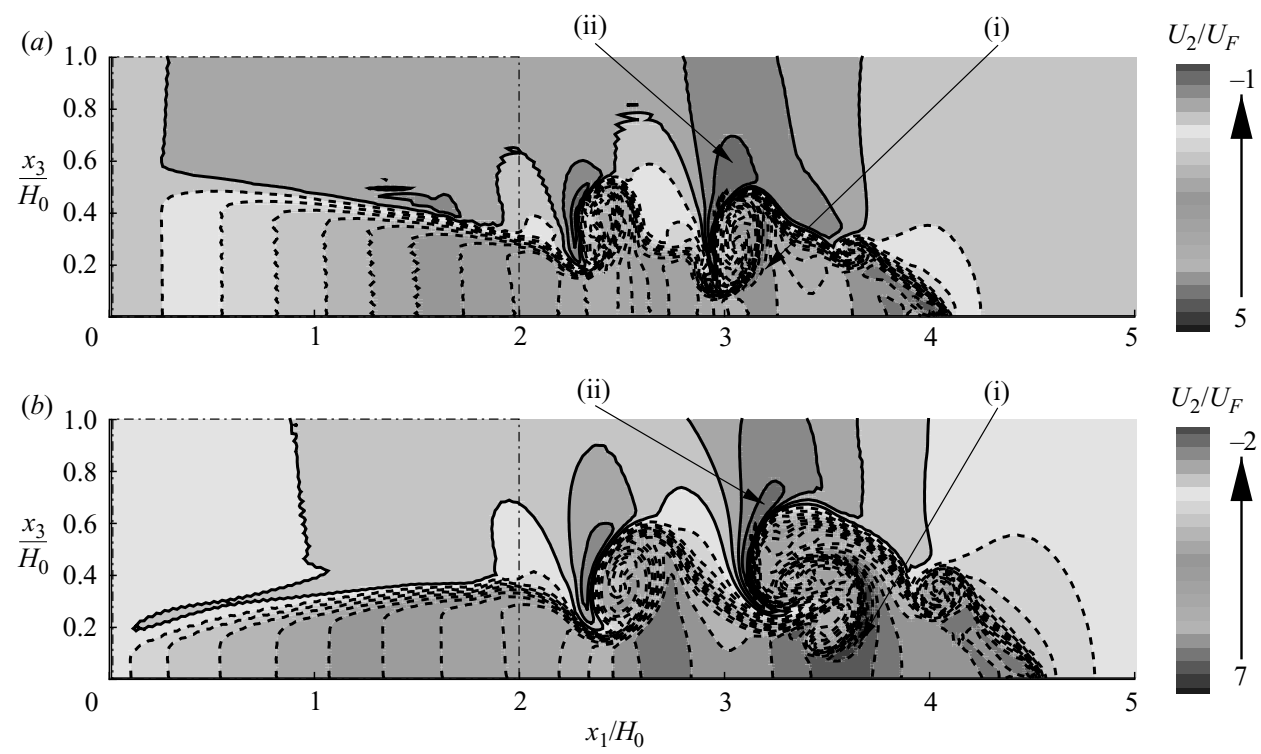

FIGURE 6. Non-hydrostatic numerical simulations of the two-dimensional inviscid density current, showing contour plots of the transverse velocity $U_{2}$ and of the interface positions. (a) Transverse velocity $U_{2}$ of a gravity current for slope $\sigma=0$ and $(b) \sigma=\pi / 2$. The contour levels are $(a)-1 \leqslant U_{2} / U_{F} \leqslant 5$ by increments of 0.3 and $(b)-2 \leqslant U_{2} / U_{F} \leqslant 7$ in increments of 0.45. Point (i) is the location of maximum transverse velocity inside the current and point (ii) is the location of maximum transverse velocity outside of it. The parameters used in the simulations are: $H_{0} / D=1 / 5, f t_{F}=2.25, H_{0} / R_{0}=1 / 2, L_{R} / R_{0}=0.33$. The dash-dot line indicates the location of the gravity current at $t=0$. Note the non-hydrostatic velocity field outside the current. 
does not occur at the foremost leading position of the front. As the dynamics shows, the magnitude of the transverse velocity $U_{2}$ is higher for gravity current fronts on sloping surfaces than fronts on horizontal surfaces. Note that for $\sigma=0$, the maximum transverse velocity $U_{2}$ is of the order of +5 of the front speed $U_{F}$ inside the current and -1 outside of it. The latter results from the non-hydrostatic velocity field. These results are also in accord with the theory of $\S \S 2.1 .3$ and 2.2.3.

\section{Dynamics of axisymmetric gravity currents under the shallow-water approximation}

3.1. Numerical simulation of whallow-water equations with 'wetted' surface

\subsubsection{Scaling of variables}

As explained in $\S 2$ and Appendix A, the front propagation velocity in a nonrotating fluid may be scaled (this scaling follows from the balance of the horizontal pressure gradient and inertia term) as

$$
\frac{\mathrm{d} R}{\mathrm{~d} t}=U_{F_{0}}=C_{1}\left(2 H g^{\prime}\right)^{1 / 2},
$$

where the constant $C_{1}$ is of order unity, and it takes into account the specific geometry of the current and bottom friction (e.g. Rottman \& Simpson 1983). The conservation of mass for an axisymmetric gravity current (assuming a constant similar form of the current, which $\S 2$ showed is approximately valid) gives

$$
H_{0} R_{0}^{2}=H R^{2} .
$$

Integrating (3.1) and using (3.2) leads to

$$
\frac{\left(R^{2}-R_{0}^{2}\right)}{R_{0}^{2}}=\frac{2 C_{1} t\left(2 g^{\prime} H_{0}\right)^{1 / 2}}{R_{0}} .
$$

The front propagation velocity $U_{F}$ in a rotating fluid is lower than that in the non-rotating flow, but when $f t \leqslant 1$ is of the same order (see $\S 2$ ). Thence,

$$
U_{F}=\frac{\mathrm{d} R}{\mathrm{~d} t}=U_{F_{0}} \phi\left(f t, L_{R} / R_{0}\right),
$$

where $U_{F_{0}}$ is the front velocity in a non-rotating fluid given by (3.1). Here, $\phi$ is a dimensionless function of $f t$ and $L_{R} / R_{0}$ that depends on the dynamics of the whole current and on local processes of the flow. From (3.2)-(3.4), we obtain

$$
\begin{aligned}
R^{* 2}=\frac{\left(R^{2} / R_{0}^{2}-1\right)}{2 C_{1}\left(L_{R} / R_{0}\right)} & =\int_{0}^{f t} \phi\left(\tau, L_{R} / R_{0}\right) \mathrm{d} \tau \\
& =\Phi\left(f t, L_{R} / R_{0}\right) .
\end{aligned}
$$

\subsubsection{Computational model}

In a second set of numerical calculations, the evolution of the current is studied when $f t \gg 1$ using the shallow-water approximation with small level of frictional losses corresponding to very high Reynolds numbers. In order to avoid imposing a front condition, the domain boundary is sufficiently long to contain the gravity current and a thin layer of fluid on the horizontal surface (wetted surface) is considered. The speeds of propagation in our simulations were scaled following the balance of horizontal pressure gradient and inertial terms through a constant $C_{1}$ (see Mahalov et al. 2000). An analysis is given in Appendix A.2 of how such a front oscillates. 
Shallow-water theory was for example shown by Rottman \& Simpson (1983) and Bonnecaze et al. (1995) to describe well the the evolution of gravity currents without rotation. This approach differs from Bonnecaze et al. (1995) and Ungarish \& Huppert (1998) who used the same condition at the front as for non-rotating flow. Note that Mahalov et al. (2000) focused on the details of the front only for times up to about $3 f^{-1}$, so that oscillatory effects were not considered.

The two-dimensional shallow-water equations are of the form of St Venant equations with constant Coriolis parameter $f$. These are taken as the model of a shallow layer of turbulent fluid. In using these equations we assume that: $(a)$ fluid is incompressible; $(b)$ the gravity current is created by the release of a liquid into an ambient fluid; $(c)$ vertical acceleration is negligible; $(d)$ shear stresses effects are negligible at the free surface; $(e)$ bottom slope is small; $(f)$ friction losses are computed using phenomenological turbulent drag formulae. In our numerical simulations, we consider only one layer of shallow fluid (heavy layer), the layer interface is assumed to be the 'free-surface' in our computation and shear stresses at the interface are being neglected. If we define the following depth-averaged quantities

$$
u_{1}=\frac{1}{H} \int_{z_{b}}^{H+z_{b}} U_{1} \mathrm{~d} z, u_{2}=\frac{1}{H} \int_{z_{b}}^{H+z_{b}} U_{2} \mathrm{~d} z,
$$

the two-dimensional depth-averaged equations of motion can be written as:

$$
\begin{aligned}
\frac{\partial H}{\partial t}+\nabla \cdot(H \boldsymbol{u}) & =0, \\
\frac{\partial H \boldsymbol{u}}{\partial t}+\boldsymbol{u} \cdot(\nabla H \boldsymbol{u}) & =-f \boldsymbol{e}_{z} \wedge H \boldsymbol{u}-g^{\prime} H \nabla\left(H+z_{b}\right)-\nabla H \boldsymbol{T}-\boldsymbol{T}_{b},
\end{aligned}
$$

where $\nabla=\left(\partial / x_{1}, \partial / x_{2}\right)$. Here, $H$ is the water depth, $\boldsymbol{u}=\left(u_{1}, u_{2}\right)$ represents the Cartesian depth-averaged velocity components in the $x_{1}$ - and $x_{2}$-directions, $x_{3}$ is the vertical direction, $z_{b}$ is the bottom elevation $\left(z_{b}=0\right.$ in our case), $f=2 \Omega$ is the Coriolis parameter, $t$ is the time, $g^{\prime}=g \Delta \rho / \rho_{0}$ is the reduced gravity acceleration; $\boldsymbol{T}_{b}=\left(\tau_{b x_{1}}, \tau_{b x_{2}}\right)$ are the bottom shear stress components and $\boldsymbol{T}=\left(T_{x_{1} x_{1}}, T_{x_{1} x_{2}}, T_{x_{2} x_{2}}\right)$ are the depth-averaged effective stress components. In our formulation, the effective stress components are neglected and the shear stresses on the bottom surface are approximated by using the Chezy formulae $\tau_{b x_{1}}=g^{\prime} u_{1}\left(u_{1}^{2}+u_{2}^{2}\right)^{1 / 2} / C^{2}$ and $\tau_{b x_{2}}=$ $g^{\prime} u_{2}\left(u_{1}^{2}+u_{2}^{2}\right)^{1 / 2} / C^{2}$, where $C$ is the Chezy constant (Vreugdenhill 1993). Computations were performed without friction $(C=\infty)$ and with friction, taking the standard value for a turbulent flow where $C=80$. (The frictional calculation for limited time $f t<10$ had been computed before by Mahalov et al. 2000.) We found that the front structure and current dynamics are only slightly changed if friction is included, but the magnitude is significantly reduced.

In the numerical simulations, the governing equations (3.7)-(3.8) were written in conservation form. The physical domain was transformed to a rectangle by means of a mapping technique and the numerical integration was performed using an explicit finite-difference flux-vector method (Pacheco \& Pacheco-Vega 2003). The boundaries of the domain were kept constant during the computation.

\subsubsection{Results of numerical simulations}

Numerical simulations are carried out with initial conditions satisfying $L_{R} / R_{0}=$ $0.33,0.44$ and 0.66 . The release of the fluid is from rest and with background rotation from $t=0$. The normalized numerical results for the radius are plotted in figure 7 for the frictionless condition for the initial adjustment period $(f t \leqslant 10)$ which means 


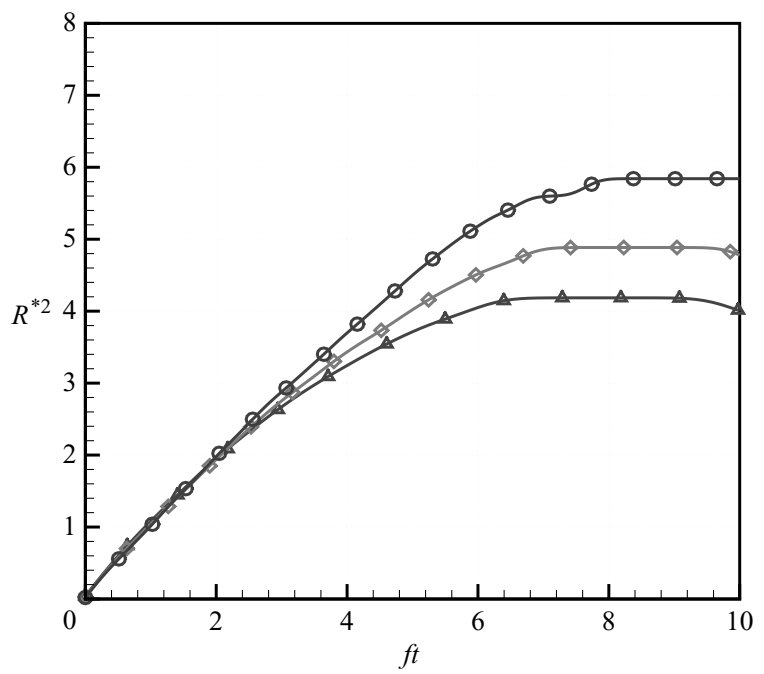

FIGURE 7. Numerical simulation of the hydrostatic development of a frictionless axisymmetric density-driven current, when $f t \sim 1$. The non-dimensional radius function $R^{* 2}$ is plotted as a function of the non-dimensional time $f t$ and different ratios $L_{R} / R_{0}\left(=B u^{1 / 2}\right): \triangle, L_{R} / R_{0}=0.33$; $\diamond, L_{R} / R_{0}=0.44 ; \bigcirc, L_{R} / R_{0}=0.66$. The rotation is applied at $t=0$.

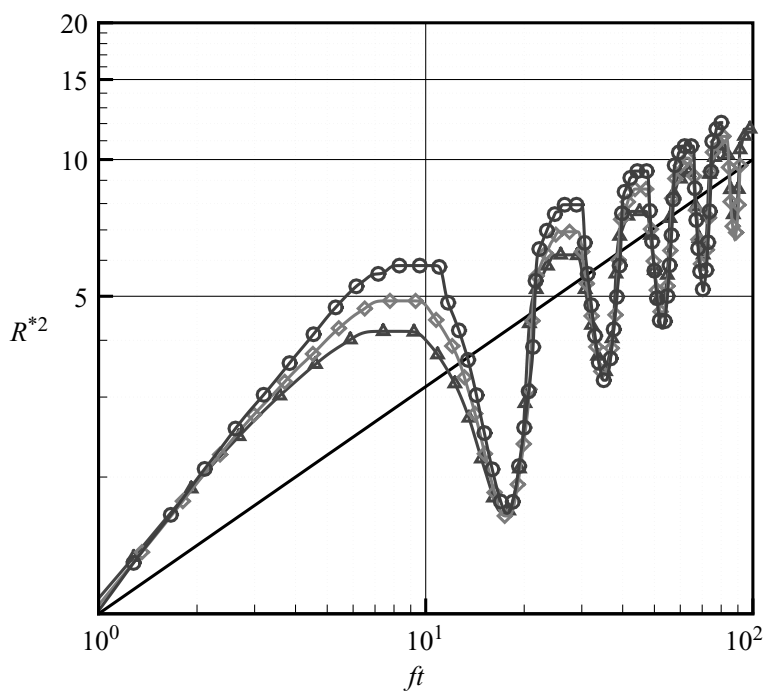

FiguRE 8. Numerical results for the hydrostatic asymptotic development of an axisymmetric density current where $f t \gg 1$. The non-dimensional radius function $R^{* 2}$ is plotted as a function of $f t: \triangle, L_{R} / R_{0}=0.33 ; \diamond, L_{R} / R_{0}=0.44 ; \bigcirc, L_{R} / R_{0}=0.66$.

less than about half a rotation period. The time to the first maximum in the recent experiment results of Hallworth et al. (2001) occurs at $f t \approx 7-9$, and compares well with the numerical simulation where $f t \approx 8-9$.

In figure 8 , the radius is presented on a log-log plot in order to compare with the asymptotic theory of $\S 3.2$. These results using the shallow-water model show that the radial velocity not only increases, but when $f t \approx 9$ for frictionless flows (which maybe an overestimate by $50 \%$, cf. figure 8 ), it reverses. So the radius stops increasing and 

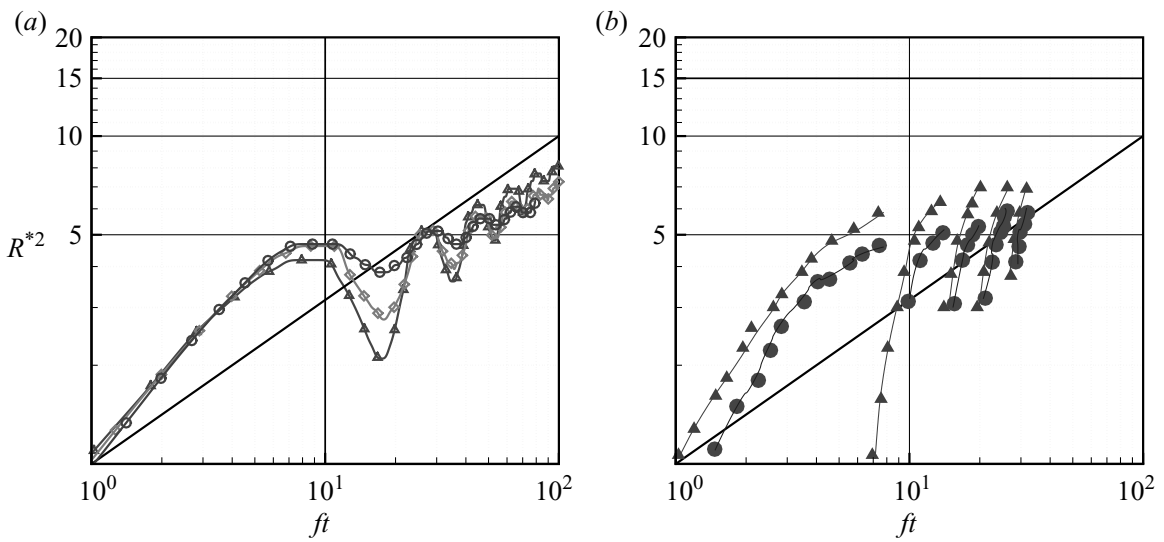

FIGURE 9. Comparison of the non-dimensional function $R^{* 2}$ when $f t \gg 1$ between the asymptotic hydrostatic numerical simulation where friction is being considered as defined by $C$, and experiments of Hallworth et al. (2001). (a) Numerical results with $C=80: \triangle, L_{R} / R_{0}=0.33$; $\diamond, L_{R} / R_{0}=0.44 ; \bigcirc, L_{R} / R_{0}=0.66$. (b) Results from experiments of Hallworth et al. (2001): $\bigcirc$, $L_{R} / R_{0} \approx 2(\mathrm{R} 11) ; \triangle, L_{R} / R_{0} \approx 3(\mathrm{R} 4)$.

begins to oscillate (whether there is friction or not). The oscillations in $R^{* 2}$ (defined by (3.5)) decrease in amplitude with time (which is expected from the linear theory of $\S 3.2$ ) with and without friction. Their period is up to $30 \%$ greater than $2 \pi / f$, i.e. one rotation period. The normalized amplitudes of oscillations are dependent on the $L_{R} / R_{0}$ ratio as given by (3.5), however, the period of oscillation is unaffected by $L_{R} / R_{0}$ for the range of values considered in our numerical simulations. In the experimental observations of Hallworth et al. (2001) for $L_{R} / R_{0} \approx 1.5-3$, their angular frequency $\omega$ was found to be about $5 \%$ higher than the inertial frequency $f=2 \Omega$.

The amplitude of oscillations for the numerical simulations with bottom friction and the experimental results of Hallworth et al. (2001) are depicted in figure 9. Without friction, the mean radius function $\left\langle R^{* 2}\right\rangle(f t)$ increases approximately in proportion to $\sqrt{f t}$, as shown in figure 8 , whereas with friction, the increase in $\left\langle R^{* 2}\right\rangle(f t)$ is reduced (see figure $9 a$ ). The experimental results shown in figure $9(b)$ correspond to the experiments labeled as $\mathrm{R} 4\left(L_{R} / R_{0} \approx 3\right)$ and $\mathrm{R} 11\left(L_{R} / R_{0} \approx 2\right)$ of Hallworth et al. (2001) normalized to accord with (3.5). The amplitude of oscillations of our numerical results when friction was considered, is approximately of the same order as the experiments of Hallworth et al. (2001).

The solution of the shallow-water equations (on a thin fluid surface layer) results in an oscillatory growth of the mean radius rather than a fixed constant asymptotic limit $R_{\infty}$ which results from inviscid front analysis on a plane (see Appendix A.1). Similar large amplitude oscillations are also observed in other simulations of axisymmetric gravity currents using a three-dimensional viscous Navier-Stokes code at a Reynolds number of about $10^{3}$. This confirms that the shallow-water simulations are valid. These results will be reported elsewhere.

The radius of the front is plotted in figure 10 for a comparison to be made with the non-hydrostatic results using the Eulerian non-hydrostatic simulation described in $\S 2.3$ for the same $L_{R} / R_{0}=0.33$ as figure 4 . The dashed line in figure 10 indicates the time where the effect of rotation begins, the dash-dot line in the same figure, indicates the time where the front stops according to the two-dimensional perturbation theory. The symbol $\nabla$ corresponds to the non-rotating case, $\bigcirc$ corresponds to the rotating 


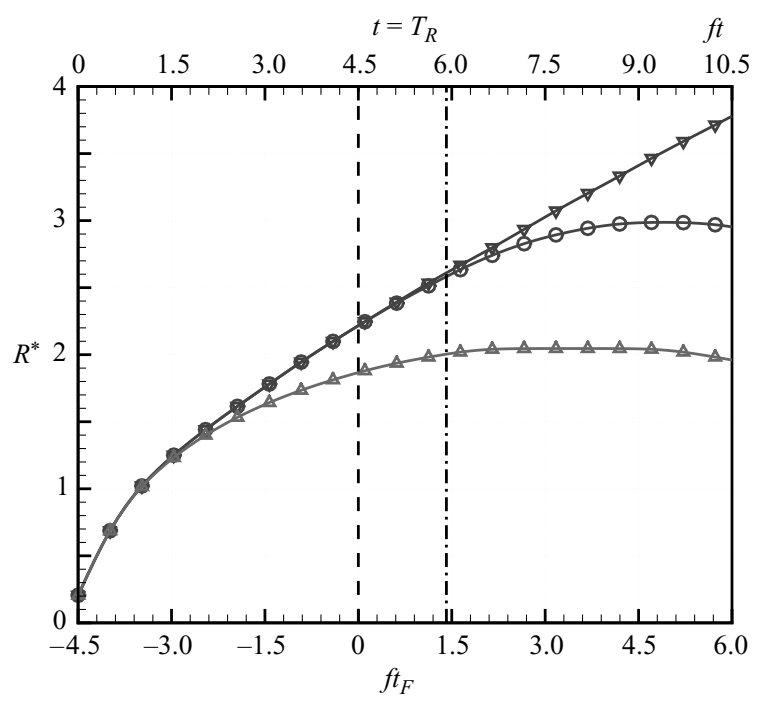

FiguRE 10. Comparison for the non-dimensional function $R^{*}$ obtained with shallow-water hydrostatic simulation without friction for $L_{R} / R_{0}=0.33$. $\nabla$, no rotation; $\bigcirc$, rotation initiated at $t=4.5 f^{-1} ; \triangle$, rotation initiated at $t=0$.
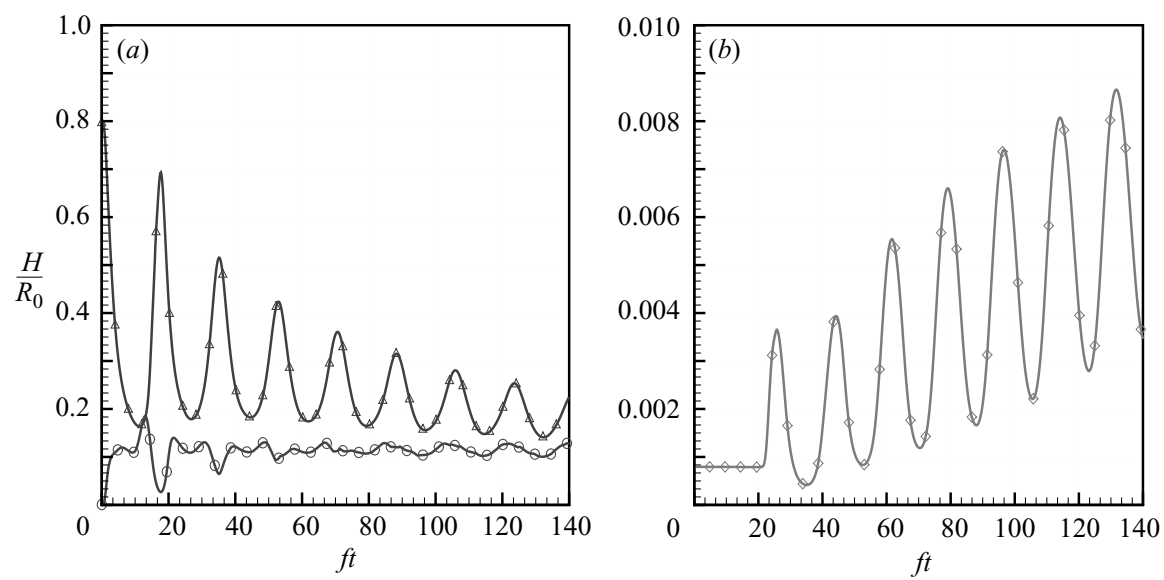

Figure 11. Computed depth motion for different fixed radial positions. The numerical conditions are: $L_{R} / R_{0}=0.33$ and Chezy constant $C=\infty$. (a) Dimensionless depth for region where motions are approximately hydrostatic. $\triangle$, corresponds to $R / R_{0}=0.5 ; O$, corresponds to $R / R_{0}=1$.6. (b) Non-dimensional height for transitional region at a fixed location $R / R_{0}=3.8$. Showing here the front location oscillates as it passes through a given point.

case when the velocity is initiated at $t=4.5 f^{-1} \mathrm{~s}$ and $\triangle$ when rotation was initiated at $t=0$. Note that the radial velocity of the front decreases at a slower pace compared to the Eulerian non-hydrostatic two-dimensional simulation. A difference would be expected from the linear theory of $\S 2$, which shows that for $f t \lesssim 1$, non-hydrostatic effects are significant.

To understand the oscillatory behaviour of the gravity current with rotation, it is necessary to examine how the depth varies with time at various radii. The result presented in figure $11(a)$ shows how in the centre of the current where $R / R_{0}=0.5$ 


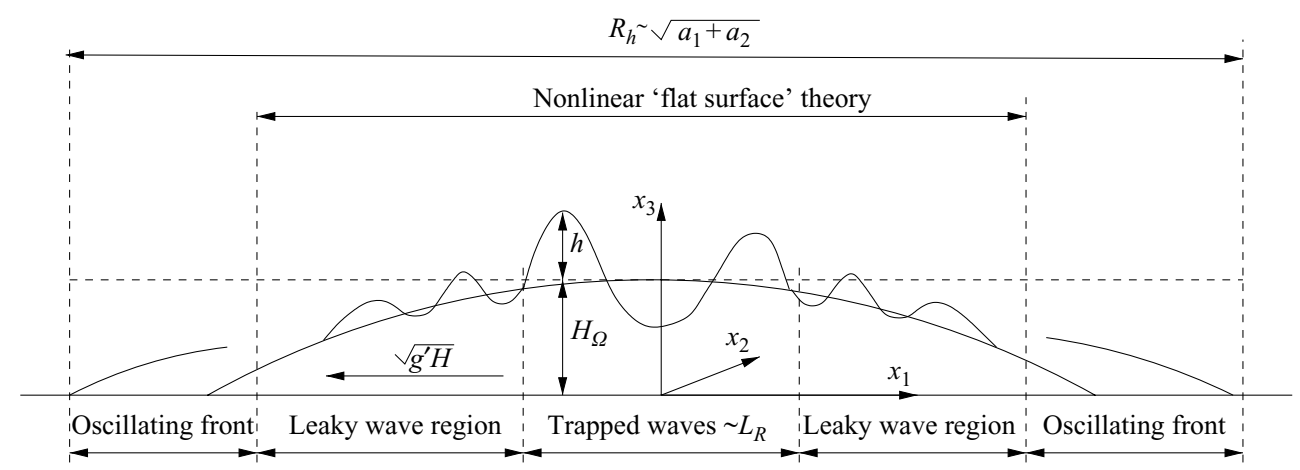

FIGURE 12. Schematic diagram for wave motion on a gravity current front, showing the different regions as energy leaks out from the centre.

and $R \sim 1.7 L_{R}$, where $L_{R}$ is the Rossby deformation radius, the depth oscillates (over a period $\sim 2 \pi / f$ ). Also the amplitude decreases slowly (by a factor $1 / e$ ) over five turnover periods. However, outside the initial radius $R_{0}$ and greater than $L_{R}$, the depth $H(R)$ rises to $1 / 8$ of $H$ at $R / R_{0}=0.5$ and then oscillates at an amplitude that slowly increases to a level comparable to that in the central region of the current (where the current was started). At a larger radius of $3.8 R_{0}$ (see figure $11 b$ ) when the front reaches this point, the oscillation amplitudes are less than $1 / 100$ of those in the centre; however they are comparable to the mean depth because the front position itself is oscillating. Over five turnover periods, their relative magnitude decreases. Here the gravity current behaves similarly to water waves approaching a shore-line. It appears from other computations not presented here, that even with strong rotation, i.e. $L_{R}<R_{0}$, a gravity current set-up within the radius $R_{0}$ oscillates as it spreads out. Initially, outside the radius $L_{R}$ the current level is quite smooth, but the oscillations leak out from the central region. When these oscillations reach the front, they cause it to be displaced. The period of all these oscillations is comparable to that of the rotation period (see figure 12).

\subsection{Oscillating solutions and asymptotics at large times}

A gravity current can be divided into three regions shown in figure 12: (i) a central region with approximately constant mean depth; (ii) a transitional region described by equations of motion for long waves in water of varying depth; and (iii) a frontal region which is characterized by non-hydrostatic effects and wave breaking. The area of the latter region shrinks with time and its influence on the overall gravity current behaviour diminishes. A local analysis of the region is included in Appendix A.2.

In the central region (i) the motions are approximately hydrostatic and the shallow-water equations with constant mean depth can be used to study the overall averaged properties of the current behaviour. The dynamics in region (ii) are similar to the behaviour of long waves on a beach analysed by Peregrine (1967). When water waves approach a beach they usually increase in amplitude and break. Equations corresponding to Boussinesq's approximation are derived in Peregrine (1967) to describe this phenomena. These are shallow-water equations with an additional dispersive term. The equations are formally valid before wave-breaking occurs. As the wave approaches region (iii) it becomes higher and steeper; then the shallow-water equations will not be valid since the wave will ultimately break. 
In this subsection we summarize published theory for the evolution of density currents in the 'trapped wave' region (of figure 12) in the context of rotating shallowwater equations (SWE) in order to extend linear theory to nonlinear oscillations when $f t \geqslant 1$. As we showed in $\S 2.2$, the density current has a low slope everywhere and the motions are approximately hydrostatic. Therefore, it is appropriate to use the shallowwater equations to study the overall averaged properties of the current behaviour. This can be evaluated systematically by considering the effects of oscillations on the density current dynamics, whose measure is the initial potential energy in relation to that of the current and is proportional to the ratio $L_{R} / R_{0}=\sqrt{B u}$ (Samah \& Thorpe 1993).

Consider the governing equations (3.7)-(3.8) and neglect the bottom friction. The height of the fluid is $H=H_{\Omega}+h$ with average depth over the layer $H_{\Omega}$. Here, $h$ is the perturbation in the level which is distributed over a distance $L_{R}$, as shown in figure 12. The initial-value problem (3.7)-(3.8) consists in calculating $\boldsymbol{u}$ and $h$ given their initial distribution at $t=t_{\Omega}$; the structure of its solutions for times $f t \geqslant 1$, was described in Babin et al. $(1997 b, c)$ and Reznik et al. (2001) using asymptotic methods.

For general initial condition for equations (3.7)-(3.8), both the fast changes due to inertia-gravity wave activity and the slow change of potential vorticity (PV) are present in the evolution of the initial disturbance (with special initial conditions, one or the other component could be absent). The problem of nonlinear geostrophic adjustment is to determine their mutual influence and interactions. The 'fast' hyperbolic field consists of both 'fast' inertia-gravity waves propagating away from the region of the initial disturbance and trapped waves (which Zeitlin, Medvedev \& Plougonven 2003, analysed for weakly nonlinear waves). The 'slow' field, characterized by potential vorticity dynamics, is in geostrophic balance at leading order, where the fast and slow components of motion are split. The fast component of motion is characterized by oscillations resulting from the unbalanced part of the flow (non-zero horizontal divergence and ageostrophic vorticity defined below). One part of the unbalanced component is radiated away in the form of outgoing fast waves; another part is captured by the potential vorticity and it oscillates without influencing its dynamics at leading order. However, this 'fast' hyperbolic component evolves on the background of the 'slow' PV component, producing significant modulation effects. The physical reason for this 'splitting' is that, for the slow components, the Lagrangian potential vorticity is conserved, while for the fast components, it consists of inertia-gravity waves, which do not carry potential vorticity. The split corresponds to the wellknown gap in the spectrum of rotating shallow-water equations between these modes. This split occurs for disturbances in both infinite and localized domains (continuous spectrum) as well as periodic and cylindrical domains (discrete spectrum). We refer to Gill (1982) for linear theory of waves on currents, to Babin et al. $(1997 b, c)$ and Reznik et al. (2001) for in-depth mathematical studies of the nonlinear waves, and to Stegner, Bouruet-Aubertot \& Pinchon (2004) for laboratory experiments.

We are interested in the long-time behaviour $(f t \geqslant 1)$ of solutions to $(3.7)-(3.8)$ for general the state of infinite two-layer fluid at $t=t_{0}$. In our expression, $t_{0}$ corresponds to the time when the initial gravity current front is approaching its final state (i.e. $t_{0} \sim f^{-1}$. To this end, let us express the potential vorticity $Q$ in terms of the vorticity $\omega=\partial u_{2} / \partial x_{1}-\partial u_{1} / \partial x_{2}$ as

$$
Q=\frac{\omega+f}{H}
$$


The potential vorticity is conserved in (3.7)-(3.8), and its equation for advection by the total horizontal velocity $\boldsymbol{u}$ takes the form

$$
\frac{\partial Q}{\partial t}+(\boldsymbol{u} \cdot \nabla) Q=0 .
$$

Linearizing the initial-value problem (3.7)-(3.8) yields

$$
\begin{gathered}
\frac{\partial u_{1}}{\partial t}-f u_{2}=-g^{\prime} \frac{\partial h}{\partial x_{1}}, \\
\frac{\partial u_{2}}{\partial t}+f u_{1}=-g^{\prime} \frac{\partial h}{\partial x_{2}}, \\
\frac{\partial h}{\partial t}+H_{\Omega} \nabla \cdot \boldsymbol{u}=0 .
\end{gathered}
$$

The aforementioned linear problem and its solutions are described in detail in Gill $(1982, \S 7.3)$. It is important to remember that linear potential vorticity modes correspond to zero-frequency eigenmodes in (3.11).

It is convenient to write (3.11) in terms of the ageostrophic variables (horizontal divergence $d=\partial u_{1} / \partial x_{1}+\partial u_{2} / \partial x_{2}$ and ageostrophic vorticity $\left.\omega_{a}=\omega-g^{\prime} \Delta h / f\right)$ in order to study its oscillating solutions, i.e.

$$
\begin{gathered}
\frac{\partial d}{\partial t}-f \omega_{a}=0, \\
\frac{\partial \omega_{a}}{\partial t}+f\left(1-L_{R}^{2} \Delta\right) d=0, \\
\frac{\partial h}{\partial t}+H_{\Omega} d=0 .
\end{gathered}
$$

Clearly, the hyperbolic system (3.12) is linear, and the ageostrophic fields $d$ and $\omega_{a}$ satisfy the linear wave equation. For example, for the field $d$ we have from (3.12),

$$
\frac{\partial^{2} d}{\partial(f t)^{2}}-L_{R}^{2} \Delta d+d=0 .
$$

The initial condition that determines the form and amplitude of the oscillations for the velocity field $\boldsymbol{u}$ and for the height field $h$ at $t=t_{\Omega}$ in (3.11), implies the initial condition for the horizontal divergence $d$ and the ageostrophic vorticity $\omega_{a}$.

The one-dimensional wave propagation in the context of linear rotating shallowwater equations (described in Gill 1982) showed that, by using the stationary phase method, the frequency of oscillations is equal to $f$, and the amplitude decays as $t \rightarrow \infty$ in proportion to $(f t)^{-1 / 2}$. This is consistent with the general interpretation of (3.11) given above.

The above results for the linear hyperbolic system (3.11) can be extended to the nonlinear rotating shallow-water equations using a van der Pol technique for fast singular oscillating limits $(f t \gg 1)$ for nonlinear hyperbolic problems (Babin et al. 1997b,c, 2002). This method leads to the ageostrophic component being explicitly solved in terms of the quasi-geostrophic component in the limit resonant equations. The corresponding solutions which are obtained with explicit error estimates based on resonances and delicate small divisor estimates describe nonlinear adjustment to geostrophic states. The ageostrophic component describes transient motions before the full solution reaches the geostrophic state. These asymptotic solutions generalize the linear inertio-gravity wave solutions described above. A qualitative description is 
given here - for mathematical analysis see Babin et al. $(1997 b, c)$ and Jones, Mahalov \& Nicolaenko (1998).

In the first step in the derivation of the fast singular oscillating limit equations, we apply a change of variables known as van der Pol transformation that eliminates the linear term in (3.7)-(3.8). The resulting equation is averaged over resonances between the fast inertio-gravity waves and the quasi-geostrophic (QG) field, and the approximation we seek is derived from the averaged equations. The averaged equations are the global envelope equations for all inertio-gravity waves; their solutions split into quasi-geostrophic (horizontally non-divergent) and ageostrophic (horizontally divergent) components. The quasi-geostrophic component is then decoupled from the ageostrophic modes by projecting it onto quasi-geostrophic modes (Babin et al. 1997a, c, Reznik et al. 2001). The resulting equation (in physical space) is called the quasi-geostrophic potential vorticity equation.

When applied to the rotating SWE, this technique decomposes the solutions of the initial-value problem into three terms. The first is obtained from the quasi-geostrophic approximation to the SWE which are solved with initial data projected onto quasigeostrophic modes. The second represents the asymptotic ageostrophic component of the flow in the limit $f t \gg 1$. It is obtained from an evolution equation coupled with the quasi-geostrophic component of the flow. This evolution equation has time- and space-dependent coefficients which depend on the QG component of the flow. The last term is the remainder and it is negligible for $f t \gg 1$. A crucial property of the dispersion law for rotating SWE is that (unlike in most nonlinear wave systems) three wave resonances are non-existent (Babin et al. 1997b).

This analysis shows when the linear theory for oscillations predicts the same frequency as for nonlinear oscillations, at least for region (i) of figure 12 for shallow layers on flat surfaces. Combining this nonlinear analysis of the 'flat' part of the current and an approximate frontal analysis (in Appendix A.2) shows that the frequency of slope oscillations is $f$. The significant wave oscillations on the current may lead to reflection of waves from the front (which are not analysed here). There seem to be no physical or mathematical reason for the generation of sub-harmonic oscillations.

\section{Conclusions}

The theoretical and computational studies presented here show how a gravity current changes as it is influenced by Coriolis forces and forms into a geostrophic front. We conclude that some concepts in the current theory and practical applications require modification. First, there is no universal tendency for a disturbed dense layer or current with horizontal scale $R_{0}$ to progress monotonically towards a state of geostrophic equilibrium. Since the Rossby deformation radius $L_{R}$ (typically $300 \mathrm{~km}$ for mid-tropospheric disturbances of depth $H \approx 3 \mathrm{~km}$ ) is of the same order as $R_{0}$, fronts generally oscillate rather than monotonically settle towards an equilibrium state. These oscillations are associated with horizontal divergence and ageostrophic vorticity and are nonlinear, although linear theory provides useful information. In the atmosphere, these oscillations may affect the local weather. Analytical models (e.g. Hoskins \& Bretherton 1972), which are based on balanced SG equations, do not model correctly the mean motion in these conditions. Because of the rapid change in the ageostrophic processes, numerical models require a resolution of the order of $H / 10$ in space and $H /\left(10 U_{F}\right)$ in time to represent the unsteady frontal processes. Our theory shows why geostrophic fronts do not have the remarkably constant structure 
of non-rotating currents where the Froude number of the front (i.e. $U_{F} /\left(g^{\prime} H\right)^{1 / 2}$ ) is close to unity in a wide range of flow situations (Simpson 1997).

Secondly, the front is only likely to have a universal form in very wide currents when $L_{R} / R_{0}$ is small. However, over a wide range of $L_{R} / R_{0}$, the slope tends to be of the order of $f / N$ when $f t \gtrsim 1$ (where $N=\sqrt{g^{\prime} / H}$ ). In this case the aspect ratio $H / R(t)$ is much less than the slope of the front. In general, the flow structure in the current depends on the initial value of $L_{R} / R_{0}$ and any unsteady forcing of the density current, e.g. by thermal convection in a cyclonic disturbance. Our numerical simulations and theory confirm the experimental results of Chia et al. (1982); Mahalov et al. (2000) and others, that the slope of the front decreases rapidly from $60^{\circ}$ when rotation is applied. The theory is consistent with geophysical estimates.

Thirdly, as a result of linear effects, the whole current and the front oscillate with frequency $f$, while nonlinear effects cause the front to continue to move forward, rather than tending to reach a fixed asymptotic position with mean radius $R_{\infty}$. The latter conclusion is consistent with the experimental results of Hallworth et al. (2001). It is broadly consistent with the observed tendency of atmospheric gravity currents to move faster when they have high fluctuations.

The numerical solution of the shallow-water equations shows the front moving forward at an average velocity proportional to $(f t)^{-1 / 2}$, which is the same time scale as for the decay of linear waves on the current. However, a nonlinear theory for this net movement of the front has not yet been developed.

In order to assess the effects of surface shear stresses, we compare the depth of the layer current $(H)$ with the thickness $\delta$ of the Ekman layer and the time scale for viscous shear effects to affect the flow. For a laminar flow, $\delta=\sqrt{v / f} \sim 10^{-3} \mathrm{~m}$. Here, $v$ is the kinematic viscosity. Then the time for viscosity to affect the geostrophic front is of order $f^{-1}(H / \delta)$. Since this decay time is clearly greater than $f^{-1}$, laminar-flow viscous effects are negligible at high Reynolds numbers. Note also that $H \gg \delta$ for most flows. In a turbulent flow where $\delta \sim 1 / 10\left(u_{*} / f\right)$, where $u_{*}(\sim|\boldsymbol{u}| / 10)$ is the 'friction' velocity, the criterion is $H \gg 10^{-2} u_{*} / f$, which is satisfied in atmospheric geostrophic fronts. (Note here that the thickness of the shear layer $\delta \sim 0.2 u_{*} / f \sim 500 \mathrm{~m}$, so that for many gravity currents in the atmosphere the shear stresses are significant.) The idealized theoretical approach was extended to examine the effects of terrain slopes. The effects of rotation are important because the analysis shows that even quite small slopes $\sigma$ (greater than the slope of the front which is very small in rotating flow) may have a large effect on anticyclonic frontal motion. This may lead to considerable mixing. By comparison, without rotation, the theory confirms laboratory experiments that the speed and the form of the gravity current front is unchanged on sloping surfaces even as large as $30^{\circ}$.

The mathematical analysis and precise numerical simulations of fronts developed here can be applied to various problems involving density currents and fronts in flows with continuous stratification. The ageostrophic processes analysed here require more detailed numerical simulations that are currently used in numerical weather predictions and oceanographic codes.

We would like to acknowledge the useful comments of Professors H. Huppert and M. Ungarish. This research was supported by the AFOSR Grant F49620-931-0172, the NSF Environmental Geochemistry and Biochemistry Initiative Grant 97-08452 and the Office of Vice President for Research. The support for J. R. P. from CONACyT, México, is also greately appreciated. J.C. R.H. and A.M. are grateful for the initial stimulus of this study from Professors B. Hoskins and K. Browning at 
Reading University and from the Isaac Newton Institue Programme on Atmosphere Ocean Dynamics held in Cambridge in 1996. J.C.R.H. acknowledges the Natural Environment Research Council's support to the centre for Polar Observation and Modelling at UCL. A. M. acknowledges the EPSRC support during his visit to the Department of Mathematics at UCL where some of this work was done.

\section{Appendix}

\section{A.1. Relating the front to the bulk structure}

Consider an axisymmetric gravity current with density $\rho+\Delta \rho$ on a plane, in a fluid of density $\rho$. It is released at $t=0$ and it has the same rotation rate $\Omega_{0}$ as the fluid, height $H_{0}$, radius $R_{0}$ as it tends to an asymptotic limit $H_{\infty}, R_{\infty}$. We note that the following conservation laws hold in a fixed non-rotating system of coordinates (assuming a simple shape of each stage so that the main features are defined by the height $H$ and the radius $R$, although, as shown in $\S 2$ there is some significant energy in slopes near the front on a scale $L_{R}$ ).

(a) Mass conservation

$$
H_{0} R_{0}^{2} \sim H R^{2} \sim H_{\infty} R_{\infty}^{2} .
$$

(b) Kelvin's theorem. The circulation around a vertical axis in an axisymmetric gravity current is unaffected by buoyancy forces, so that

$$
\Gamma_{0} \sim v_{\theta_{0}} R_{0}=\Gamma(t)\left(\sim \Omega R^{2} \sim v_{\theta} R\right)=\Gamma_{\infty}=v_{\theta_{\infty}} R_{\infty} .
$$

Note that this is consistent with potential vorticity conservation, where $\Omega \alpha H$, since from (A 1) and (A 2)

$$
\Omega \sim \frac{v_{\theta}}{R} \sim v_{\theta_{0}} \frac{H}{H_{0}} \sim \frac{\Omega_{0} H}{H_{0}} .
$$

Local analysis of the equilibrium front shows that the centripetal total radial pressure gradient $\partial p^{*} / \partial r$ is equal to the pressure gradient outside the current, $\partial p / \partial r$ plus the hydrostatic contribution, i.e.

$$
\frac{\partial p^{*}}{\partial r} \sim \frac{v_{\theta}^{2}}{R}=-g^{\prime} \alpha_{\infty}+\frac{\partial p}{\partial r},
$$

where $g^{\prime}=g \Delta \rho / \rho$ and $\partial p / \partial r=\Omega_{0}^{2} R_{\infty}$. Since $v_{\theta}=\Omega_{0} R_{\infty}+\Delta v_{\theta}$, and $f=2 \Omega_{0}$

$$
\Delta v_{\theta}=-g^{\prime} \frac{\alpha_{\infty}}{f},
$$

when $\left(\Delta v_{\theta}\right) \ll\left(\Omega_{0} R_{\infty}\right)$. This is equivalent to Margules' (1906) formula; but when $R_{\infty} \gg R_{0}$ (see below), (A 2) shows that $v_{\theta_{\infty}}=\Omega_{0} R_{0}^{2} / R_{\infty} \ll \Omega_{0} R_{0}$, so that

$$
\Delta v_{\theta}=-\Omega_{0} R_{\infty}=-\frac{g^{\prime} \alpha_{\infty}}{\Omega_{0}}=-\frac{2 g^{\prime} \alpha_{\infty}}{f}
$$

(i.e. twice the Margules limit).

We can now estimate how the front slope $\alpha_{\infty}$ relates to the overall shape of the current $H / R$, because this affects the oscillations within the current. As a measure of this relation we define a re-scaled front slope

$$
\tilde{\alpha}=\alpha_{\infty} /(H / R) \sim \frac{\Omega_{0} \Delta v_{\theta}}{g^{\prime} H_{0}} \frac{R_{\infty}}{R_{0}^{2}},
$$


where $\tilde{\alpha}$ is dimensionless. From (A 2), $\Omega_{0} R_{\infty}+\Delta v_{\theta}=\Omega_{0} R_{0}^{2} / R_{\infty}$, so that

$$
\Delta v_{\theta}=-\frac{\Omega_{0}\left(R_{\infty}^{2}-R_{0}^{2}\right)}{R_{\infty}}
$$

and from (A 3b), (A 3c),

$$
\alpha_{\infty} \sim \frac{\Omega_{0}^{2}}{g^{\prime}}\left(\frac{R_{\infty}-R_{0}}{R_{\infty}}\right)\left(R_{\infty}+R_{0}\right) .
$$
i.e.

$R_{\infty}$ is, to first approximation, determined by the local gravity current velocity $\sqrt{g^{\prime} H}$,

$$
\frac{\mathrm{d} R}{\mathrm{~d} t}=\frac{\mathrm{d} \Phi}{\mathrm{d} t} \sqrt{g^{\prime} H}=\frac{\mathrm{d} \Phi}{\mathrm{d} t}\left[\frac{g^{\prime} R_{0}^{2} H_{0}}{R^{2}}\right]^{1 / 2},
$$

where from $\S 3.1, \Phi(t)$ is an $O(1)$ variable that decays to zero on time scale $f^{-1}$. Thence,

$$
\left(\frac{R^{2}}{R_{0}^{2}}-1\right) /\left(L_{R} / R_{0}\right)=\Phi\left(t f, L_{R} / R_{0}\right) .
$$

Note that from $\S 2,3$, the radial displacement of the front, in order of magnitude terms, is

$$
\left(R_{\infty}-R_{0}\right) \sim \sqrt{g^{\prime} H_{0}} \Omega_{0}^{-1} \sim L_{R_{0}},
$$

where

$$
L_{R_{0}}=\frac{\sqrt{g^{\prime} H_{0}}}{\Omega_{0}}
$$

is the Rossby radius of the initial current.

Thence, from (A $6 c$ ) if $L_{R_{0}}^{2} \ll R_{0}^{2}, R_{\infty} \sim R_{0}$ so that from (A 5b) $\alpha_{\infty} \sim \Omega_{0}^{2} L_{R_{0}} / g^{\prime}$. Therefore,

$$
\tilde{\alpha}=\alpha_{\infty} R_{\infty} / h_{\infty} \sim R_{0} / L_{R_{0}} \gg 1 .
$$

Similarly, from (A 6c) if $L_{R_{0}}^{2} \gg R_{0}^{2}$,

$$
R_{\infty} \sim L_{R_{0}} \gg R_{0}
$$

so that $\alpha_{\infty} \sim \Omega_{0}^{2} L_{R_{0}} / g^{\prime}$ and $\tilde{\alpha} \sim\left(\Omega_{0}^{2} L_{R_{0}} / g^{\prime}\right)\left(L_{R_{0}}^{3} / H_{0} R_{0}^{2}\right) \sim L_{R_{0}}^{2} / R_{0}^{2} \gg 1$. Thus, in both cases $\tilde{\alpha} \gg 1$, so that the slope of the front (if it reaches equilibrium) is greater than the average slope of the current $\left(\sim H_{\infty} / R_{\infty}\right)$, but less than the initial slope of $(\pi / 3)$. Thus, since the front undergoes a substantial change on a time scale of order $f^{-1}$, oscillations must develop. Numerical simulations show that these oscillations cause the ultimate value of $R_{\infty}$ to be larger than the estimates given in (A $6 c$ ) and (A 7b). Simulations using a Navier-Stokes code $\left(R e \sim 10^{3}\right)$ show that in the slow decay to the asymptotic state, slopes in the interior of the current are greater than those of the front edge.

\section{A.2. Oscillations of the front}

We develop a simple linear analysis to indicate how the front oscillates. Consider the asymptotic front perturbed by a small angle $\alpha^{\prime}$ at $t=0$, but which is larger than the very small asymptotic value $\alpha_{\infty}$. It follows that

$$
-\frac{\partial p^{\prime}}{\partial x}=g^{\prime} \alpha^{\prime} \quad \text { at } \quad t=0
$$


over the distance where the slope changes, which is of order $L_{R_{0}}$. Assume an oscillation develops where the front is moving outwards, i.e. $\partial u^{\prime} / \partial t>0$, because $\partial v^{\prime} / \partial t=-f u^{\prime}$, so that $v<0$, and thence $u<0$. A model equation for small perturbations is

$$
\begin{aligned}
& \frac{\partial u^{\prime}}{\partial t}-f v^{\prime}=-\frac{\partial p^{\prime}}{\partial x}=g^{\prime} \alpha^{\prime}(t), \\
& \frac{\partial v^{\prime}}{\partial t}+f u^{\prime}=0 .
\end{aligned}
$$

Thence, if $\alpha^{\prime}(t)$ is a step function, $\alpha^{\prime}(t)=\alpha_{o} H(t)$,

$$
\frac{\partial^{2} u^{\prime}}{\partial t^{2}}+f^{2} u^{\prime}=g^{\prime} \alpha_{o} \delta(t)
$$

with a solution

$$
u^{\prime}=\frac{g^{\prime} \alpha^{\prime}}{f}(\sin (f t)) H(t),
$$

This frequency is independent of $L_{R} / R$ as observed in experiments. As the surface adjusts to maintain constant mass, a rarefaction wave travels back along the front, a positive wave travels forward, etc. Note that if the wavelength of these waves is $\lambda=\tilde{\lambda} R \sim L_{R}$, then resonance occurs if their frequency is equal to the frequency of the front oscillations $f$. The frequency $c / \lambda$ of oscillation in the central region $\left(\omega_{c}\right)$ is

$$
\omega_{c} \sim \frac{c}{\lambda}=\frac{\sqrt{g^{\prime} H}}{\tilde{\lambda} R} \sim f \quad \text { or } \quad \frac{L_{R}}{R} \sim \tilde{\lambda} .
$$

Thus, large oscillations occur if $L_{R_{0}} / R_{0} \sim 1$, whereas small oscillations will be present when $L_{R_{0}} / R_{0} \ll 1$. Note that in Hallworth et al. (2001) experimental results $\omega \simeq f$ for $L_{R_{0}} / R_{0} \gg 1$. Our numerical simulations for rotation dominated flows $\left(L_{R_{0}} / R_{0} \ll 1\right)$ yield approximately the same frequency $\omega \simeq f$. In both cases resonant like oscillations occur coupling the flow in regions (i), (ii) and (iii) of figure 12.

\section{REFERENCES}

AtKinson, B. W. 1981 Dynamical Meteorology: an Introductory Selection. Methuen.

Babin, A., Mahalov, A. \& Nicolaenko, B. 1997a Global regularity and integrability of 3D Euler and Navier-Stokes equations for uniformly rotating fluids. Asymp. Anal. 15, 103-150.

Babin, A., Mahalov, A. \& Nicolaenko, B. $1997 b$ Global splitting and regularity of rotating shallow-water equations. Eur. J. Mech. B/ Fluids 16, 725-754.

Babin, A., Mahalov, A. \& Nicolaenko, B. 1997c Regularity and integrability of rotating shallowwater equations. In C. R. Acad. Sci. Paris, pp. 593-598.

Babin, A., Mahalov, A. \& Nicolaenko, B. 1998 On nonlinear baroclinic waves and adjustment of pancake dynamics. Theor. Comput. Fluid Dyn. 11, 215-235.

Babin, A., Mahalov, A. \& Nicolaenko, B. 2002 Fast Singular Oscillating Limits of Stably Stratified Three-Dimensional Euler-Boussinesq Equations and Ageostrophic Wave fronts. Cambridge University Press.

Benjamin, T. B. 1968 Gravity currents and related phenomena. J. Fluid Mech. 31, 209-248.

Bonnecaze, R. T., Hallworth, M. A., Huppert, H. E. \& Lister, J. R. 1995 Axi-symmetric particle-driven gravity currents. J. Fluid Mech. 294, 93-121.

Britter, J. E. \& Linden, P. F. 1980 The motion of the front of a gravity current travelling down an incline. J. Fluid Mech. 99, 531-543.

Britter, J. E. \& Simpson, J. E. 1978 Experiments on the dynamics of a gravity current head. J. Fluid Mech. 88, 223-240.

Charney, J. G. 1948 On the scale of atmospheric motions. Geofys. Publ. Oslo 17, 1-17. 
Chen, R., Boyer, D. L. \& TAO, L. 1993 Laboratory simulation of atmospheric motions in the vicinity of Antarctica. J. Atmos. Sci. 50, 4058-4079.

Chia, F., Griffiths, R. W. \& Linden, P. F. 1982 Laboratory experiments on fronts. Part ii: The formation of cyclonic eddies at upwelling fronts. Geophys. Astrophys. Fluid Dyn. 19, 189-206.

Creswell, G. R. \& Golding, T. G. 1986 Observations of south flowing currents in the southwestern Indian ocean. Deep-Sea Res. 27, 449-466.

Cullen, M. J. P. 2002 New mathematical developments in atmosphere and ocean dynamics and their application to computer simulations. In Large Scale Atmosphere Ocean Dynamics (ed. J. Norbury \& I. Roulstone). Cambridge University Press.

Embid, P. F. \& MaJdA, A. J. 1996 Averaging over fast gravity waves for geophysical flows with arbitrary potential vorticity. Commun. Part. Diff. Eqn. 21, 619-658.

Gill, A. E. 1982 Atmosphere-Ocean Dynamics. Academic.

Hallworth, M. A., Huppert, H. E. \& Ungarish, M. 2001 Axisymmetric gravity currents in a rotating system: experimental and numerical investigations. J. Fluid Mech. 447, 1-29.

Haltiner, G. J. \& Williams, R. T. 1980 Numerical Prediction and Dynamic Meteorology. John Wiley.

Hoskins, B. J. \& Bretherton, F. P. 1972 Atmospheric frontogenesis models: mathematical formulation and solution. J. Atmos. Sci. 29, 11-37.

IvEY, G. N. 1987 Boundary mixing in a rotating stratified fluid. J. Fluid Mech. 183, 25-44.

Jones, D. A., Mahalov, A. \& Nicolaenko, B. 1998 A numerical study of an operator splitting method for rotating flows with large ageostrophic initial data. Theoret. Comput. Fluid Dyn. 13, 143-159.

von Kármán, T. 1940 The engineer graples with nonlinear problems. Bull. Am. Math. Soc. 46, 615-683.

Legeckis, R. \& Creswell, G. 1981 Satellite observations of sea surface temperature off the coast of western and southern Australia. Deep-Sea Res. 28, 279-306.

Lentz, S. J. \& HelfRich, K. R. 2002 Buoyant gravity current along a sloping bottom in a rotating fluid. J. Fluid Mech. 464, 251-278.

Linden, P. F. \& Simpson, J. E. 1989 Self-similar solutions of the shallow-water equations representing gravity current with variable inflow. J. Fluid Mech. 202, 1-16.

MaAs, L. R. M. \& van Haren, J. J. M. 1987 Observations on the vertical structure of tidal and inertial currents in central North Sea. J. Mar. Res. 45, 293-318.

McWilliams, J. C. 1985 Submesoscale coherent vortices in the ocean. Rev. Geophys. 23, 165-254.

Mahalov, A., Pacheco, J. R., Voropayev, S. I., Fernando, H. J. S. \& Hunt, J. C. R. 2000 Effects of rotation on fronts of density currents. Phys. Letters A 270, 149-156.

Manins, P. C. \& Sawford, B. L. 1979 Katabatic winds - field case-study. Q. J. R. Met. Soc. 105, 1011-1025.

Margules, M. 1906 Über Temperaturschichtung in stationär bewegter und ruhender Luft. Met. Z. 23, $165-254$.

Milne-Thompson, L. M. 1968 Theoretical Hydrodynamics. Macmillan.

NoH, Y. \& Fernando, H. J. S. 1992 The motion of a buoyant cloud along an incline in the presence of boundary mixing. J. Fluid Mech. 235, 557-577.

Noh, Y. \& Fernando, H. J. S. 1993 A numerical model of the fluid motion at a density front in the presence of background turbulence. J. Phys. Oceanogr. 23, 1142-1153.

Norbury, J. \& Roulstone, I. 2002 Large Scale Ocean Dynamics. Cambridge University Press.

Pacheco, J. R. 2001 The solution of viscous incompressible jet flows using non-staggered boundary fitted coordinate methods. Intl J. Numer. Meth. Fluids 35, 71-91.

Pacheco, J. R. \& Pacheco-Vega, A. 2003 Analysis of thin film flows using a flux vector splitting. J. Fluids Engng. 125, 365-374.

Pacheco, J. R. \& Peck, R. E. 2000 Non-staggered grid boundary-fitted coordinate method for free surface flows. Numer. Heat Transfer B 37, 267-291.

PARKeR, D. J. 2000 Frontal theory. Weather 55, 120-134.

Peregrine, D. H. 1967 Long waves on a beach. J. Fluid Mech. 27, 815-827.

QiU, B. \& Imasato, N. 1988 Baroclinic instability of buoyancy driven coastal density current. J. Geophys. Res. 93, 5037-5050. 
Reznik, G. M., Zeitlin, V. \& Jelloul, M. B. 2001 Nonlinear theory of geostrophic adjustment. Part 1. Rotating shallow-water model. J. Fluid Mech. 445, 93-120.

Rottman, J. W., Hunt, J. C. R. \& Mercer, A. 1985 The initial and gravity spreading phases of heavy gas dispersion: comparison of models with Phase I data. J. Hazard. Mat. 11, 261-279.

RotTman, J. W. \& Simpson, J. E. 1983 Gravity currents produced by instantaneous releases of a heavy fluid in a rectangular channel. J. Fluid Mech. 135, 95-110.

Rotunno, R. 1983 On the linear theory of the land and sea breeze. J. Atmos. Sci. 40, 1999-2009.

SAmah, A. A. \& Thorpe, A. J. 1993 Gravity wave propagation in a frontal environment. In Waves and Turbulence in Stably Stratified Flows (ed. S. D. Morh \& J. C. King). Clarendon.

SidI, J. \& BARAT, J. 1986 Observational evidence of an inertial wind structure in the stratosphere. J. Geophys. Res. 91, 1209-1217.

Simpson, J. E. 1997 Gravity Currents. Cambridge University Press.

Stegner, A., Bouruet-Aubertot, P. \& Pinchon, T. 2004 Nonlinear adjustment of density fronts. Part 1. The Rossby scenario and the experimental reality. J. Fluid Mech. 502, 335-360.

Turner, J. S. 1973 Buoyancy Effects in Fluids. Cambridge University Press.

Ungarish, M. \& Huppert, H. E. 1998 The effects of rotation on axisymmetric gravity currents. J. Fluid Mech. 362, 17-51.

VReugdenhill, C. B. 1993 Numerical Methods for Shallow-Water Flows. Kluwer.

Westerweel, J., Hofmann, T., Fukushima, C. \& Hunt, J. C. R. 2002 The turbulent/non-turbulent interface at the outer boundary of a self-similar turbulent jet. Exps. Fluids 33, 873-878.

Zang, Y., Street, R. I. \& KosefF, J. R. 1994 A non-staggered grid, fractional step method for time dependent incompressible Navier-Stokes equations in curvilinear coordinates. J. Comput. Phys. 114, 18-33.

Zeitlin, V., Medvedev, S. B. \& Plougonven, R. 2003 Frontal geostrophic adjustment, slow manifold and nonlinear wave phenomena in one-dimensional rotating shallow water. Part 1 . Theory. J. Fluid Mech. 481, 269-290.

ZuKosKi, E. E. 1966 Influence of viscosity, surface terrain, and inclination angle on motion of long bubbles in closed tubes. J. Fluid Mech. 25, 821-837. 